\title{
Asymmetric flux models for particle-size segregation in granular avalanches
}

\author{
P. Gajjar ${ }^{1, \dagger}$ and J. M. N. T. Gray ${ }^{1}$ \\ ${ }^{1}$ School of Mathematics and Manchester Centre for Nonlinear Dynamics, \\ University of Manchester, Manchester M13 9PL, UK
}

(Received 4 April 2014; revised 25 July 2014; accepted 23 August 2014)

Particle-size segregation commonly occurs in both wet and dry granular free-surface flows through the combined processes of kinetic sieving and squeeze expulsion. As the granular material is sheared downslope, the particle matrix dilates slightly and small grains tend to percolate down through the gaps, because they are more likely than the large grains to fit into the available space. Larger particles are then levered upwards in order to maintain an almost uniform solids volume fraction through the depth. Recent experimental observations suggest that a single small particle can percolate downwards through a matrix of large particles faster than a large particle can be levered upwards through a matrix of fines. In this paper, this effect is modelled by using a flux function that is asymmetric about its maximum point, differing from the symmetric quadratic form used in recent models of particle-size segregation. For illustration, a cubic flux function is examined in this paper, which can be either a convex or a non-convex function of the small-particle concentration. The method of characteristics is used to derive exact steady-state solutions for non-diffuse segregation in two dimensions, with an inflow concentration that is (i) homogeneous and (ii) normally graded, with small particles above the large. As well as generating shocks and expansion fans, the new asymmetric flux function generates semi-shocks, which have characteristics intersecting with the shock just from one side. In the absence of diffusive remixing, these can significantly enhance the distance over which complete segregation occurs.

Key words: granular materials, mixing, pattern formation

\section{Introduction}

Granular avalanches are dense shallow flows of grains down an incline, which may be continuous or intermittent (Pudasaini \& Hutter 2007). As well as occurring in chute flows (Savage \& Hutter 1989; Gray, Wieland \& Hutter 1999; Khakhar, McCarthy \& Ottino 1999; Pouliquen 1999), avalanches are an integral component of more complex granular flows that involve static or slowly moving regions, such as in hoppers, heaps and rotating drums (Nityanand, Manley \& Henein 1986; Gray 2001; GDR MiDi 2004; Fan et al. 2012, 2014). There is a wide body of literature modelling avalanches, which makes the following assumptions: (i) the granular material is an incompressible continuum (Savage \& Hutter 1989; Hutter, Wang \&

$\dagger$ Email address for correspondence: parmesh.gajjar@alumni.manchester.ac.uk 
Pudasaini 2005); (ii) the transient and constantly evolving force chains only act over short distances and are well approximated by a lithostatic pressure balance (Pitman et al. 2003; Rognon et al. 2007); and (iii) the solids volume fraction $\Phi$ is constant in the range 0.49-0.64 (Silbert et al. 2001; Kumaran 2006, 2008), a slight dilation from a static arrangement of random close-packed spheres (Berryman 1983). These three assumptions are also good approximations for bidisperse and polydisperse flows (Rognon et al. 2007; Tripathi \& Khakhar 2011; Marks, Rognon \& Einav 2012). Avalanches are notoriously good at sorting particles by size. Not only can this lead to a rich variety of patterns in flowing mixtures arrested by shock waves or basal deposition (e.g. Williams 1976; Gray \& Hutter 1997; Hill et al. 1999; Ottino \& Khakhar 2000), but also it can be problematic in a wide range of sectors, from agriculture and food manufacturing, to mining, bulk chemical processing and pharmaceutical production (e.g. Dyer 1929; Johanson 1978; Shinbrot \& Muzzio 2000; Marks et al. 2013). Segregation also occurs in many granular flows in our natural environment, including snow avalanches (Savage \& Hutter 1989; Bartelt \& McArdell 2009), debris flows (Iverson 1997; Stock \& Dietrich 2006), pyroclastic surges (Calder, Sparks \& Gardeweg 2000) and subaqueous grain flows (Dingler \& Anima 1987), which create a diverse array of sedimentary deposits (Branney \& Kokelaar 1992) as well as subtle feedback effects that can enhance flow run-out (Iverson \& Vallance 2001; Johnson et al. 2012; Kokelaar et al. 2014).

The dominant segregation mechanisms in granular avalanches are widely thought to be those of kinetic sieving (Middleton 1970) and squeeze expulsion (Savage \& Lun 1988). As the grains avalanche downslope, adjacent layers of grains are sheared over one another, and the volume fraction is slightly dilated. This creates a random fluctuating sieve in which smaller grains preferentially fall down through gaps that open up beneath them, because they are statistically more likely than the larger grains to fit into the available space (Savage \& Lun 1988). The solids volume fraction in monodisperse avalanches is almost constant with depth (Silbert et al. 2001) and this is also true in bidisperse systems (Rognon et al. 2007). As a result, there has to be a return flow of large particles towards the free surface due to force imbalances, which Savage \& Lun (1988) termed 'squeeze expulsion'. The combination of kinetic sieving and squeeze expulsion is a highly efficient mechanism for sorting, with even slight differences in the size ratio causing the grains to separate into inversely graded layers, with large particles lying on top of the fines. This upward coarsening of the particle-size distribution is also referred to as 'reverse grading' (e.g. Schminck 1967; Fisher \& Mattinson 1968; Sohn \& Chough 1993).

Gray \& Thornton (2005) derived a bidisperse continuum model for segregation using mixture theory (e.g Morland 1992), with the segregation dependent on a redistribution of partial pressures. The fundamental idea behind this model is that larger particles support a greater proportion of the lithostatic overburden pressure as the small particles percolate downwards. This is consistent with the kinetic sieving idea, but Golick \& Daniels (2009) suggested that the physical reason for increased forces may simply be that the large grains have significantly more contacts than the fines, and are, therefore, more likely to be part of force chain networks. In standard mixture theory, coarse grains would support a volume-fraction-weighted proportion of the pressure, in order to balance the gravitational force pulling them downwards. However, because they support a larger fraction of the overburden pressure, the pressure gradient pushing them upwards is slightly bigger than the gravitational force pulling them downwards, which produces a net upward force that drives them towards the surface. Conversely, the gravitational force acting on the small particles is larger 
than the pressure gradient that they experience, and hence they percolate downwards. The perturbations of the pressure away from the volume-fraction-weighted lithostatic pressure distribution in standard mixture theory are therefore crucial for segregation.

When either of the large or small particles are in a pure phase, they must carry all of the load, and the pressure perturbation must be zero for no net motion. Gray $\&$ Thornton (2005) postulated a quadratic concentration dependence for the pressure perturbations, with zero perturbation at 0 and $100 \%$ concentration of particles. This yields a flux function that is symmetric about $50 \%$ concentration. When this model is combined with diffusive remixing of the particles (Gray \& Chugunov 2006), it is able to quantitatively match experimental measurements of segregation in chute flow experiments (Savage \& Lun 1988; Dolgunin \& Ukolov 1995; Vallance \& Savage 2000; Wiederseiner et al. 2011) as well as discrete element simulations of segregation in periodic boxes (Thornton et al. 2012). It has also been successfully extended to include interstitial fluid (Thornton, Gray \& Hogg 2006), particle deposition (Gray \& Ancey 2009), non-lithostatic pressure (Fan \& Hill 2011), depth averaging (Gray \& Kokelaar 2010b,a; Woodhouse et al. 2012) and polydisperse distributions with discrete grain-size classes (Gray \& Ancey 2011) as well as a continuous spectrum of particle sizes (Marks et al. 2012).

Although the quadratic model has proved to be very effective at modelling segregation, Golick \& Daniels (2009) observed, in their annular ring shear cell experiments, that a small particle falls through a matrix of large particles faster (figure $1 a$ ) than a large particle rises through a matrix of small particles (figure $1 b$ ). Equivalently, a single small particle is more likely to find a percolation path through a matrix of large grains, than a single large grain is able to push the fines out of the way as it is squeezed upwards. It is important to stress from the outset that this asymmetry in the segregation velocities is still consistent with the mass balance of both species, because the differential segregation rates occur at different concentrations. Several authors have found asymmetric segregation rates by modelling the segregation velocity with a dependence on the shear strain rate (May et al. 2010a; May, Shearer \& Daniels 2010b; Marks \& Einav 2011). As these models only include a symmetric quadratic concentration dependence, the local asymmetry in the segregation rates is independent of the local particle concentration, and only dependent on the flow height. In some flows, however, the shear strain rate can be approximated by a constant (GDR MiDi 2004; May et al. 2010b; Gray \& Ancey 2011), and so asymmetric segregation rates must instead be a result of the local particle concentration. This paper examines the impact of the local particle concentration by modelling the non-reciprocity of the segregation rates using a concentration-dependent flux function that is asymmetric about its maximum point.

The asymmetry can be achieved by using higher-order flux functions, such as cubic or quartic ones, which harks back to some of the early work on segregation by Bridgwater, Foo \& Stephens (1985), who directly postulated a cubic form of the flux function with a repeated root when the small-particle concentration equalled $100 \%$. Savage \& Lun's (1988) information entropy approach also yielded an even more complex nonlinear concentration dependence. The asymmetric flux functions studied here can either be (i) convex or (ii) non-convex, with a single inflection point that occurs at high small-particle concentrations. The non-convex case implies that the maximum large-particle velocity occurs when there are several large particles in close proximity to each other (figure 1c) as opposed to the convex case where the maximum rise rate occurs for a single particle on its own (figure $1 b$ ). This is the segregation equivalent of the well-known sedimentation problem (Kynch 1952; Rhee, 
(a)

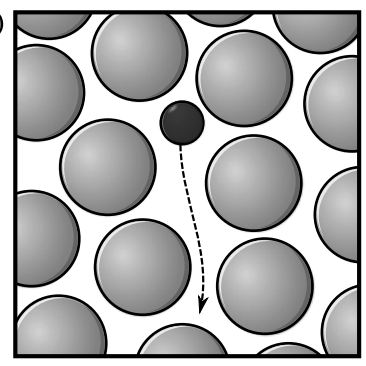

(b)

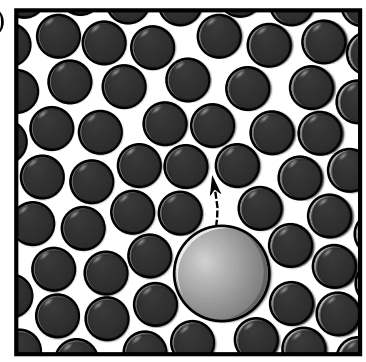

(c)

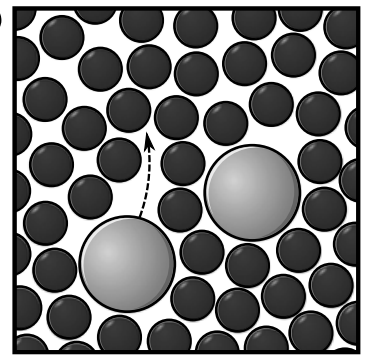

FIGURE 1. Experimental observations (Golick \& Daniels 2009) suggest that a single small particle $(a)$ percolates downwards through a matrix of large particles at a faster rate than a single large particle $(b)$ rises through a matrix of small particles. This asymmetry at the extremes of local concentration can be modelled by an asymmetric segregation flux, which can be either convex or non-convex, with an inflection point at high small-particle concentrations (see $\S 3$ ). In the latter case, a single large particle $(b)$ will rise towards the surface slower than an intermediate concentration when there are several large particles (c) rising at the same time.

Aris \& Amundson 1986) in which two particles settle under gravity in a viscous fluid faster than a single particle alone (Batchelor 1972). Similar mathematical problems also occur in traffic flow (Lighthill \& Whitham 1955), where the velocity of cars tends to zero very rapidly as the road approaches maximum density. In order to model the asymmetric behaviour in these environments, a number of flux functions have been proposed, including logarithmic, cubic and quartic forms (Greenberg 1959; Shannon, Stroupe \& Tory 1963). In this paper, the mixture theory derivation of the segregation equation is extended to the case of asymmetric flux functions and the implications for the homogeneous and normally graded inflow problems of Gray \& Thornton (2005) and Thornton et al. (2006) are investigated.

\section{The governing segregation equation}

\subsection{Mixture theory}

Consider a bidisperse mixture of large $(l)$ and small $(s)$ particles of the same density flowing down a slope inclined at an angle $\zeta$ to the horizontal. As shown in figure 2, a coordinate system $O x y z$ is defined with the $x$ coordinate pointing down the slope, the $y$ coordinate pointing horizontally across the plane and the $z$ coordinate being the upward-pointing normal. Following Gray \& Thornton (2005), the interstitial fluid is neglected. This implicitly assumes that the solids volume fraction is constant and uniform throughout the mixture (Rognon et al. 2007) and that the density of the interstitial fluid is incorporated into the density of the grains (Thornton et al. 2006). In this framework, each constituent $(v=l, s)$ occupies a local volume fraction $\phi^{v}$ per unit granular volume (Morland 1992) and the sum of the local volume fractions is equal to unity,

$$
\phi^{l}+\phi^{s}=1 .
$$

Each constituent has a partial density $\rho^{v}$, a partial velocity $\boldsymbol{u}^{v}$ and a partial pressure $p^{\nu}$. These are defined per unit volume of the mixture, and are related to the intrinsic properties of the constituents, defined per unit volume of the constituent, by

$$
\rho^{v}=\phi^{v} \rho^{\nu *}, \quad \boldsymbol{u}^{\nu}=\boldsymbol{u}^{\nu *}, \quad p^{v}=\phi^{v} p^{\nu *},
$$




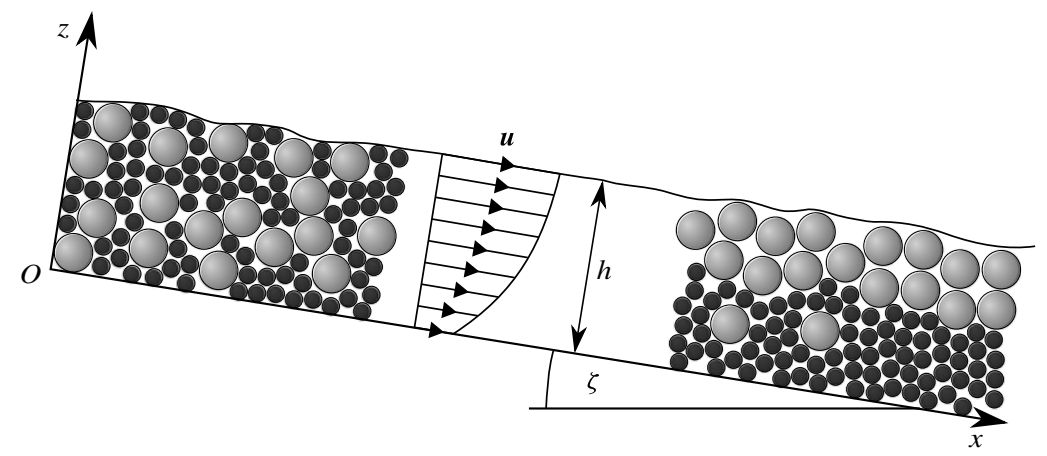

FIgURE 2. A coordinate system Oxyz is defined with the $x$-axis pointing down the plane, the $y$-axis horizontally across the plane (pointing into the page) and the $z$-axis being the upward-pointing normal. The slope is inclined at an angle $\zeta$ to the horizontal, $h$ is the flow depth and $\boldsymbol{u}$ is the bulk velocity, which generally includes both shear and basal slip. From a homogeneously mixed inflow at the origin, the particles segregate into inversely graded layers, with large particles overlying fines. With asymmetric segregation models, the distance for complete segregation is dependent on the inflow concentration and can be significantly longer than the quadratic flux case.

where a starred quantity denotes an intrinsic property. The bulk density, bulk velocity and bulk pressure of the flow are defined as

$$
\rho=\rho^{l}+\rho^{s}, \quad \rho \boldsymbol{u}=\rho^{l} \boldsymbol{u}^{l}+\rho^{s} \boldsymbol{u}^{s}, \quad p=p^{l}+p^{s},
$$

where the partial velocity $\boldsymbol{u}^{\nu}$ and the bulk velocity $\boldsymbol{u}$ have components $\left(u^{v}, v^{v}, w^{v}\right)$ and $(u, v, w)$ in the $(x, y, z)$ directions, respectively.

Each constituent satisfies its own mass conservation law,

$$
\frac{\partial \rho^{v}}{\partial t}+\nabla \cdot\left(\rho^{v} \boldsymbol{u}^{v}\right)=0 \quad(v=l, s),
$$

where $\nabla$ is the standard vector differential operator 'del' and - is the dot product. The bulk mass balance is obtained by summing (2.4) over both constituents. Since the intrinsic densities of the two phases are assumed to be equal, it follows from (2.1)-(2.4) that the bulk velocity field is incompressible, i.e. $\boldsymbol{\nabla} \cdot \boldsymbol{u}=0$. Each constituent also satisfies its own momentum conservation law,

$$
\frac{\partial}{\partial t}\left(\rho^{v} \boldsymbol{u}^{v}\right)+\nabla \cdot\left(\rho^{v} \boldsymbol{u}^{v} \otimes \boldsymbol{u}^{v}\right)=-\nabla p^{v}+\rho^{v} \boldsymbol{g}+\boldsymbol{\beta}^{v} \quad(v=l, s),
$$

where $\otimes$ denotes the dyadic product, $\boldsymbol{g}$ is the gravitational force and $\boldsymbol{\beta}^{v}$ is the interaction force exerted on constituent $v$ by the other constituent. By Newton's third law, these interaction forces are equal and opposite, $\boldsymbol{\beta}^{l}=-\boldsymbol{\beta}^{s}$.

\subsection{Normal momentum balance}

It is assumed that the flow is shallow, i.e. the depth of the flow is much less than its length, so that the normal acceleration terms in the constituent momentum balances (2.5) may be neglected. The individual constituent momentum balances may be summed to give the bulk normal momentum balance,

$$
0=-\frac{\partial p}{\partial z}-\rho g \cos \zeta
$$


By assuming the pressure to be zero at the free surface $z=h,(2.6)$ can be integrated to show that the bulk pressure field is lithostatic,

$$
p=\rho g(h-z) \cos \zeta .
$$

Following Gray \& Thornton (2005), each constituent is assumed to support a proportion of the bulk lithostatic pressure,

$$
p^{s}=f^{s} p, \quad p^{l}=f^{l} p,
$$

where $(2.3 \mathrm{c})$ implies that the scaling factors $f^{v}$ must sum to unity,

$$
f^{l}+f^{s}=1 .
$$

Each constituent cannot support negative pressure, so $f^{v}$ lies in the range $0 \leqslant f^{v} \leqslant 1$.

Following Gray \& Thornton (2005) and Gray \& Chugunov (2006), the interaction drag $\boldsymbol{\beta}^{v}$ in the constituent momentum balance (2.5) is assumed to take the form

$$
\boldsymbol{\beta}^{v}=p \nabla f^{v}-\rho^{v} c\left(\boldsymbol{u}^{v}-\boldsymbol{u}\right)-\rho d \nabla \phi^{v} \quad(v=l, s) .
$$

The first term on the right-hand side combines with the partial pressure gradient term in (2.5) to ensure that the particles are driven by intrinsic pressure gradients rather than partial ones. The second term is a linear drag between constituent $v$ and the bulk particle matrix, and has coefficient $c$. Finally, the third term represents the diffusive remixing, whose strength is given by the diffusion coefficient $d$. It models the random walks that particles experience as they are sheared in the flow (Dolgunin \& Ukolov 1995; Gray \& Chugunov 2006). With the interaction drag (2.10) and the lithostatic pressure distribution (2.7), the momentum balance (2.5) implies that the normal particle flux of constituent $v$ is

$$
\phi^{v} w^{v}=\phi^{v} w+\left(f^{v}-\phi^{v}\right) \frac{g}{c} \cos \zeta-\frac{d}{c} \frac{\partial \phi^{v}}{\partial z} \quad(v=l, s),
$$

where the first term on the right-hand side gives the normal advection due to the bulk velocity, the second term gives the segregation flux and the last term gives the flux due to diffusive remixing. The factor $f^{v}-\phi^{v}$ controls whether a particle will rise or fall due to segregation, and is essentially the proportion by which the pressure deviates away from the volume-fraction-weighted average. In the absence of diffusion, large particles carry excess pressure and will therefore rise, while small grains carry less of the overburden and fall relative to the bulk. In general, the factors $f^{\nu}-\phi^{v}$ are functions of the large- and small-particle concentrations, $\phi^{l}$ and $\phi^{s}$ (see e.g. Gray \& Ancey 2011), but in the bidisperse case $\phi^{l}=1-\phi^{s}$ from (2.1) and thus $f^{\nu}-\phi^{\nu}$ can be written solely in terms of the small-particle concentration $\phi^{s}$ as

$$
\left.\begin{array}{l}
f^{l}-\phi^{l}=+b F\left(\phi^{s}\right), \\
f^{s}-\phi^{s}=-b F\left(\phi^{s}\right),
\end{array}\right\}
$$

where the function $F$ describes the dependence of the segregation on the small-particle concentration $\phi^{s}$, and $b$ is a parameter that is dependent on the shear rate, the local pressure, the grain-size ratio and possibly other variables. The opposite signs in (2.12) ensure that the segregation fluxes are equal and opposite for either constituent, which preserves the global mass balance, i.e. summing (2.11) over both constituents, 
(a)

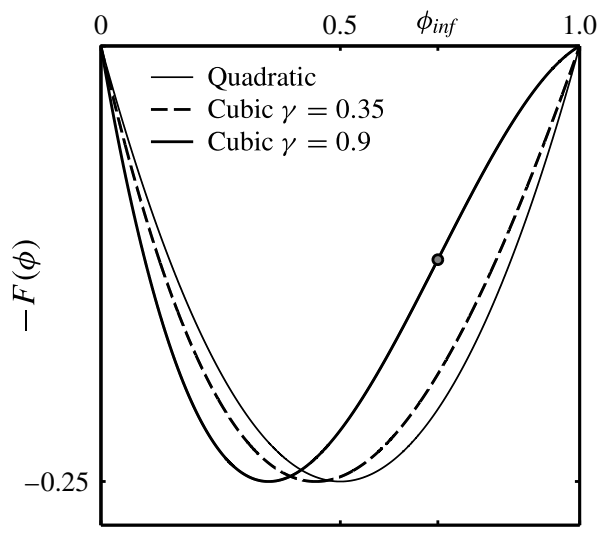

(b)

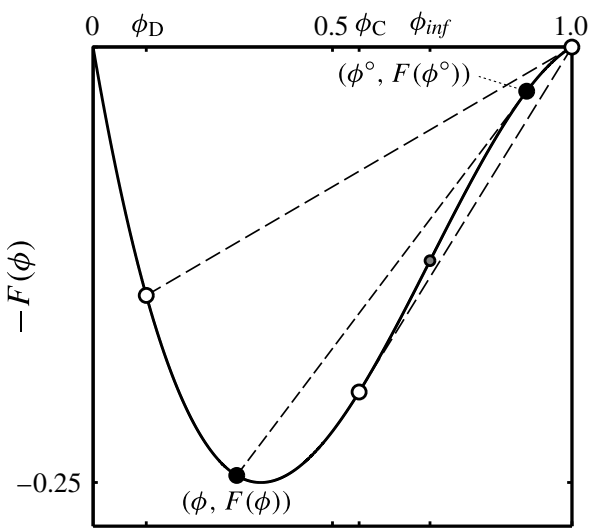

FIgURE 3. (a) The simple cubic function (3.4) demonstrates the behaviour of the entire class of asymmetric functions, with parameter $\gamma$ in the range $0<\gamma \leqslant 1$. For small amounts of asymmetry $(\gamma=0.35$, thick dashed line), the flux function remains convex, but larger amounts of asymmetry $(\gamma=0.90$, thick solid line) causes the flux function to be non-convex, with a single inflection point at $\phi_{\text {inf }}$ (grey circle). The symmetric quadratic flux function (2.14) is shown for comparison (thin solid line) and corresponds to $\gamma \rightarrow 0$. (b) Non-convex flux functions yield a special feature in the exact solution known as a semi-shock. A semi-shock is formed between two points $\phi$ and $\phi^{\circ}$ (black circles), where the chord between the two points is tangential to the flux curve $F$ at $\phi^{\circ}$. There are two pairs of points $\left\{1, \phi_{\mathrm{C}}\right\}$ and $\left\{\phi_{\mathrm{D}}, 1\right\}$ (open circles) that are of particular importance in the construction of the exact solution. The chord between $\phi_{\mathrm{C}}$ and $\phi=1$ is tangential to the flux curve at $\phi_{\mathrm{C}}$, whilst the chord linking $\phi_{\mathrm{D}}$ and $\phi=1$ is tangential to the flux curve at $\phi=1$. Negative values of the flux function, $-F(\phi)$, are plotted since the gradients then correspond to the gradients in the exact solution, as explained in $\S 4$.

using (2.1) and (2.12), implies that $\sum_{v=l, s} \phi^{\nu} w^{v}=w$, which is consistent with (2.3b). For large particles to rise, $F$ must be positive, and, in order that there is no net motion whenever the particles are in a pure phase, it must satisfy the constraints

$$
\left.\begin{array}{ll}
F=0, & \phi^{s}=0, \\
F=0, & \phi^{s}=1 .
\end{array}\right\}
$$

Gray \& Thornton (2005) postulated a quadratic flux function,

$$
F\left(\phi^{s}\right)=\phi^{s}\left(1-\phi^{s}\right)
$$

which is symmetric about the maximum, $F\left(\phi_{\max }\right)=1 / 4$ at $\phi_{\max }=1 / 2$, as shown in figure 3. This is the simplest function that satisfies the constraints (2.13) and it appears to capture the leading-order behaviour observed in experiments quite well (Wiederseiner et al. 2011). In this paper, more general asymmetric flux functions are investigated to understand some of the more subtle physical effects that are observed.

\subsection{The segregation equation}

The constituent velocities in the down- and cross-slope directions are assumed to be equal to the bulk velocity,

$$
u^{v}=u, \quad v^{v}=v .
$$


Substituting the particle velocities (2.11) and (2.15), together with the segregation flux (2.12), into the constituent mass balance (2.4) implies that the segregation-remixing equation for small particles is

$$
\frac{\partial \phi^{s}}{\partial t}+\nabla \cdot\left(\phi^{s} \boldsymbol{u}\right)+\frac{\partial}{\partial z}\left(-q F\left(\phi^{s}\right)\right)=\frac{\partial}{\partial z}\left(D \frac{\partial \phi^{s}}{\partial z}\right),
$$

where $q=(b / c) g \cos \zeta$ is the maximum segregation velocity, and $D=d / c$ is the diffusivity. The first term is the time rate of change of the concentration, the second term is the local advection by the bulk flow, the third term drives the particle-size segregation, whilst the fourth term accounts for diffusive remixing.

The avalanche thickness $H$ is typically much less than the downslope length scale $L$. Incompressibility of the bulk flow implies that, if $U$ is a typical downslope velocity, typical normal velocities will be of magnitude $\varepsilon U$, where $\varepsilon=H / L \ll 1$ is the aspect ratio of the avalanche. This suggests introducing non-dimensional variables, denoted by the tildes, using the scalings

$$
(x, y, z)=L(\tilde{x}, \tilde{y}, \varepsilon \tilde{z}), \quad(u, v, w)=U(\tilde{u}, \tilde{v}, \varepsilon \tilde{w}), \quad t=(L / U) \tilde{t} .
$$

Dropping the tildes, the non-dimensional segregation equation (2.16) therefore becomes

$$
\frac{\partial \phi}{\partial t}+\nabla \cdot(\phi \boldsymbol{u})+\frac{\partial}{\partial z}\left(-S_{r} F(\phi)\right)=\frac{\partial}{\partial z}\left(D_{r} \frac{\partial \phi}{\partial z}\right),
$$

where for simplicity $\phi$, without the superscript ' $s$ ', is the small-particle concentration. The large-particle concentration is $1-\phi$ from (2.1). The non-dimensional segregation and diffusive-remixing numbers are

$$
S_{r}=\frac{q L}{H U}, \quad D_{r}=\frac{D L}{H^{2} U},
$$

respectively, and their ratio, $S_{r} / D_{r}$, is known as a Péclet number $P e$ for segregation. It quantifies the strength of the segregation compared to diffusive remixing within the flow. While diffusion is very important on steep slopes or when the particle-size differences are small, it is useful to consider the non-diffuse limit in which $D_{r}=0$, because it allows considerable insight to be gained. Such conditions develop on low-inclination slopes with large size differences between the particles (Gray \& Hutter 1997; Dasgupta \& Manna 2011), where Péclet numbers exceeding 10 have been measured in experiments (Gray \& Chugunov 2006; Wiederseiner et al. 2011). In the remainder of this paper, the segregation equation (2.18) is therefore considered in the non-diffuse limit, which reduces it to

$$
\frac{\partial \phi}{\partial t}+\nabla \cdot(\phi \boldsymbol{u})+\frac{\partial}{\partial z}\left(-S_{r} F(\phi)\right)=0 .
$$

In the absence of erosion and deposition, (2.20) is solved subject to a no-flux condition at the surface and base of the avalanche, $z=s(x, t)$ and $z=b(x, t)$, respectively (see e.g. Gray \& Ancey 2011). When $D_{r}=0$, these boundary conditions reduce to

$$
F(\phi)=0 \quad \text { at } z=s(x, t) \text { and } z=b(x, t) .
$$

Since, by definition (2.13), the flux $F(\phi)$ is zero when either of the particles are in a pure phase, the no-flux condition (2.21) is satisfied when either $\phi=0$ or $\phi=1$. 


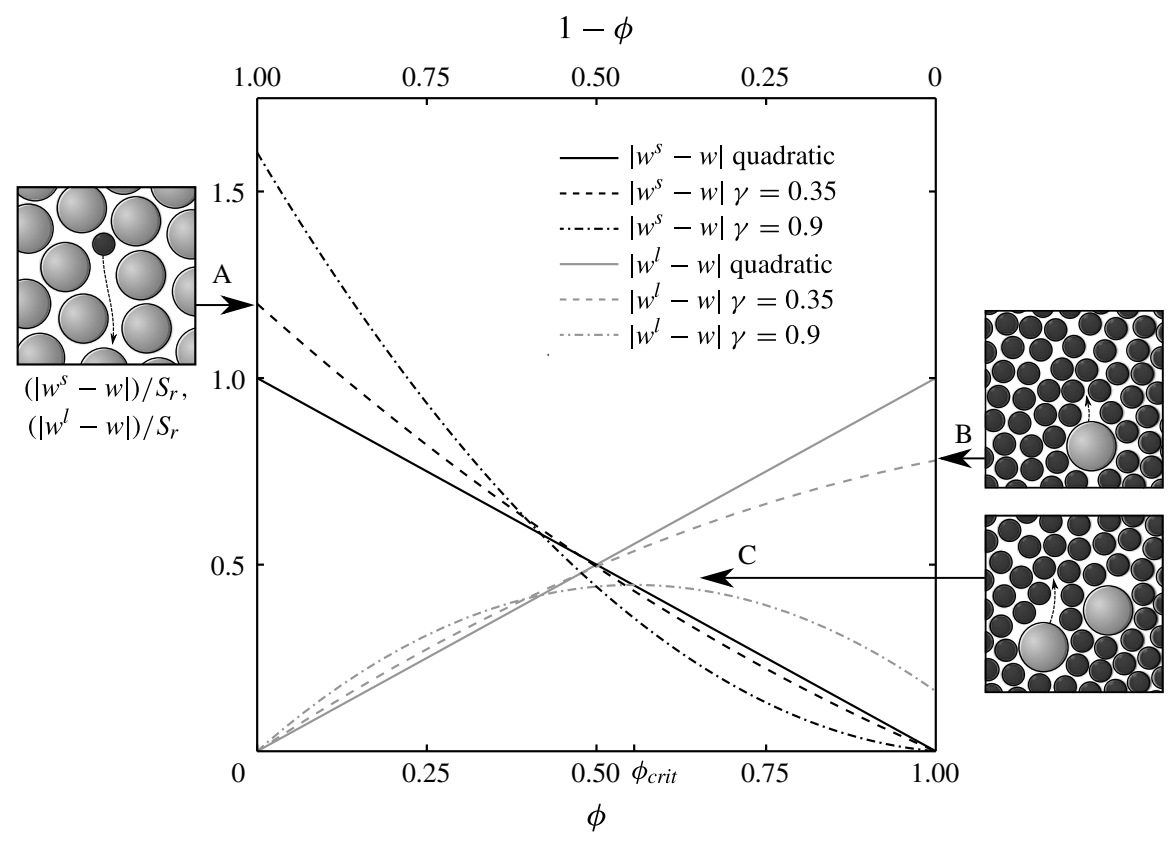

FIGURE 4. The modulus of the large (grey) and small (black) normal segregation velocities, relative to the bulk velocity $w$, plotted as a function of the small-particle concentration $\phi$ (lower axis) and large-particle concentration $1-\phi$ (upper axis). The velocities for large and small particles with the quadratic flux function (2.14) are straight (solid) lines, which attain the same maximum speed at $\phi=1$ and $\phi=0$, respectively. An asymmetric flux function is required to model the observation that small particles (A) can percolate through a matrix of large particles at a faster rate than a large particle (B) rises up through a matrix of small particles, e.g. for the convex cubic flux function (3.4) with $\gamma=0.35$ (dashed lines). For asymmetric non-convex flux functions (e.g. $\gamma=0.9$, dash-dotted lines), the maximum large-particle rise velocity occurs at $\phi_{c r i t}$, which implies that a group of large particles (C) may rise faster than an isolated large grain.

\section{Asymmetric flux functions}

In the absence of diffusion, the large- and small-particle segregation velocities in the normal direction can be derived by substituting (2.12) into (2.11) and using the scalings (2.17) to give

$$
w^{l}(\phi)=w+S_{r} \frac{F(\phi)}{1-\phi}, \quad w^{s}(\phi)=w-S_{r} \frac{F(\phi)}{\phi} .
$$

For the quadratic flux function (2.14), proposed by Gray \& Thornton (2005), this implies that, relative to the bulk flow, the normal segregation velocities are linear functions of the small-particle concentration, i.e.

$$
w^{l}(\phi)=w+S_{r} \phi, \quad w^{s}(\phi)=w-S_{r}(1-\phi) .
$$

Figure 4 shows the rise/fall rate of large/small particles (grey/black solid lines) for the quadratic flux (3.2) as a function of the small-particle concentration $\phi$. The maximum rise velocity of the large particles is $S_{r}$ at $\phi=1$ (i.e. in the limit of $100 \%$ small 
particles and $0 \%$ large particles), whilst the small particles percolate downwards at a maximum speed $-S_{r}$ when $\phi=0$ (i.e. in the limit of $0 \%$ small particles and $100 \%$ large particles). The segregation speed $\left|w^{v}-w\right|$ of each species $(v=l, s)$ has the same linear behaviour when plotted against the concentration of that species $\phi^{v}$ (where $\phi^{s}=\phi$ and $\phi^{l}=1-\phi$ from (2.1)). Critically, there is no asymmetry in the maximum rise/fall rates, as the maximum speed of small particles falling is the same as the maximum speed at which large particles rise.

In order to capture the effect that a single small particle falls through a matrix of large particles at a faster rate than a single large particle rises through a matrix of fines, asymmetric flux functions $F(\phi)$ are considered. These are skewed towards $\phi=0$, with a maximum occurring at $0<\phi_{\max }<1 / 2$, as shown in figure 3 . The flux functions are normalised so that they all have the same maximum as the quadratic flux function (2.14). For asymmetric convex flux functions, with no inflection points, the maximum small-particle percolation velocity (figure 4-A) still occurs when $\phi=0$, as in the quadratic case, but it is faster than the maximum rate at which large particles are squeezed up to the surface at $\phi=1$ (figure 4-B). In addition, for singly non-convex flux functions with an inflection point at $\phi_{\text {inf }}$ in the interval $\left(\phi_{\max }, 1\right)$, the maximum large-particle rise velocity occurs at $\phi_{\text {crit }} \neq 1$, as shown in figure $4(C)$, which satisfies

$$
\left(1-\phi_{\text {crit }}\right) F^{\prime}\left(\phi_{\text {crit }}\right)+F\left(\phi_{\text {crit }}\right)=0,
$$

where $F^{\prime}$ is the first derivative, $\mathrm{d} F / \mathrm{d} \phi$. Functions with inflection points are, therefore, also able to capture the observation that a group of large particles will sometimes rise faster than an isolated large particle on its own.

The behaviour of an entire class of asymmetric flux functions is illustrated in this paper by a simple one-parameter cubic function,

$$
F(\phi)=A_{\gamma} \phi(1-\phi)(1-\gamma \phi),
$$

where $\gamma \in(0,1]$ is a parameter that controls the amount of asymmetry and hence the non-reciprocity of the segregation rates, as shown in figures $3(a)$ and 4 . The constant $A_{\gamma}$ is chosen so that the maximum, $F\left(\phi_{\max }\right)=1 / 4$, is the same as for the quadratic case, whilst $\gamma \leqslant 1$ ensures that $F$ is positive for $\phi \in[0,1]$. In the limit $\gamma \rightarrow 0$, the quadratic flux function is recovered. For $\gamma \leqslant 0.5$, the flux function $-S_{r} F$ in (2.18) remains convex (i.e. $-S_{r} F^{\prime \prime}(\phi)>0$ for all $\phi \in[0,1]$ ), but, when $\gamma>0.5$, the flux function becomes non-convex, with a single inflection point $\phi_{\text {inf }}$ at

$$
\phi_{i n f}=\frac{1+\gamma}{3 \gamma},
$$

where $\gamma \in(0.5,1]$ means that $2 / 3 \leqslant \phi_{\text {inf }} \leqslant 1$. The rise/fall rates for the convex flux functions $(\gamma=0.35$, dashed lines) and non-convex flux functions $(\gamma=0.9$, dash-dotted lines) are shown in figure 4 . It can be seen that the rise/fall rates $\left|w^{\nu}-w\right|$ of large/small particles $(v=l, s)$ are close to linear at low concentrations of large/small particles $\phi^{\nu}$, but there is nonlinear behaviour at large $\phi^{\nu}$. Some evidence of this nonlinearity is provided by the discrete element method (DEM) simulations of Fan et al. (2014). All the results in this paper are derived for a general flux function $F$ with a maximum skewed towards low concentrations of fines. The specific cubic form (3.4) is used to illustrate the results in a practical example. Further experiments and DEM simulations will need to be performed to determine the precise form of the flux function. 
For the non-convex case, it is useful to define the image point $\phi^{\circ}$ of a concentration $\phi$ as the point where the gradient of the tangent to the flux function $F^{\prime}\left(\phi^{\circ}\right)$ is equal to the gradient of the chord joining $\phi$ to $\phi^{\circ}$ on $F$, i.e. the point $\phi^{\circ}$ satisfies

$$
F^{\prime}\left(\phi^{\circ}\right)=\frac{F(\phi)-F\left(\phi^{\circ}\right)}{\phi-\phi^{\circ}} .
$$

Comparing (3.3) with (3.6) implies that $\phi_{c r i t}$ is the image point of unity, i.e. $\phi_{c r i t}=1^{\circ}$, which is also defined as $\phi_{\mathrm{C}}$ in figure $3(b)$. For the cubic flux (3.4), the image point of $\phi$ is

$$
\phi^{\circ}=\frac{1}{2}\left(\frac{1+\gamma}{\gamma}-\phi\right) .
$$

The chord joining one such pair $\left\{\phi, \phi^{\circ}\right\}$ is shown by the black circles in figure $3(b)$. In particular, the image point of $\phi=1$ is $\phi_{\mathrm{C}}=\phi_{c r i t}$, which for the cubic function gives

$$
1^{\circ}=\phi_{\mathrm{C}}=\phi_{c r i t}=\frac{1}{2 \gamma},
$$

whereas $\phi=1$ is the image point of

$$
\phi_{\mathrm{D}}=\frac{1-\gamma}{\gamma}, \quad \text { so } \phi_{\mathrm{D}}^{\circ}=1 .
$$

The chords joining $\left\{1, \phi_{\mathrm{C}}\right\}$ and $\left\{\phi_{\mathrm{D}}, 1\right\}$ are shown by dashed lines in figure $3(b)$.

\section{The method of characteristics}

It is assumed that the depth is constant and that the transverse and normal components of the bulk velocity are zero everywhere, $v=0, w=0$. Incompressibility then implies that the downslope velocity $u=u(z)$ is only dependent on $z$, i.e. the flow is steady and uniform in $x$ (Pouliquen 1999; Silbert et al. 2001; Rognon et al. 2007; Forterre \& Pouliquen 2008). This downslope velocity profile could, for instance, be the well-known Bagnold-like profile (Bagnold 1954; GDR MiDi 2004; Jop, Forterre \& Pouliquen 2005; Gray \& Edwards 2014), or a velocity profile with both shear and basal slip such as that sketched in figure 2. Scaling the height with the flow thickness implies that $z \in[0,1]$. For steady-state non-diffuse solutions, the segregation equation (2.20) reduces to

$$
\frac{\partial}{\partial x}(\phi u(z))+\frac{\partial}{\partial z}\left(-S_{r} F(\phi)\right)=0 .
$$

By making the change of variables

$$
\xi=x, \quad \psi=\int_{0}^{z} u(\hat{z}) \mathrm{d} \hat{z},
$$

the steady-state segregation equation (4.1) is mapped into a velocity-independent form

$$
\frac{\partial \phi}{\partial \xi}+\frac{\partial}{\partial \psi}\left(-S_{r} F(\phi)\right)=0,
$$

provided $u(z) \neq 0$. This coordinate transformation was first introduced by Gray \& Thornton (2005) and is equivalent to solving the problem in streamfunction coordinates (e.g. Gray \& Ancey 2009). By virtue of the velocity scaling (2.17), the free surface that lies at $z=1$ can be mapped to $\psi=1$, without loss of generality. 
Transformation (4.2) is valid for any velocity profile $u=u(z)$, and so the inverse transformation allows the solution in the streamfunction domain $(\xi, \psi)$ to be mapped back to physical space $(x, z)$ for any velocity profile $u=u(z)$. The conservative equation (4.3) can be written in quasi-linear form as

$$
\frac{\partial \phi}{\partial \xi}-S_{r} F^{\prime}(\phi) \frac{\partial \phi}{\partial \psi}=0,
$$

where a prime denotes a derivative. This is a scalar conservation law, which can be solved by the method of characteristics (e.g. Courant \& Hilbert 1962; Whitham 1974; Rhee et al. 1986). The concentration $\phi$ is constant along characteristic curves, which may be written in the parametric form $(\xi(s), \psi(s))$, where $s$ is the distance along the curve. As the concentration $\phi$ is constant, the total derivative is zero, so

$$
\frac{\mathrm{d} \phi}{\mathrm{d} s}=\frac{\mathrm{d} \xi}{\mathrm{d} s} \frac{\partial \phi}{\partial \xi}+\frac{\mathrm{d} \psi}{\mathrm{d} s} \frac{\partial \phi}{\partial \psi}=0 .
$$

By comparing (4.4) with (4.5) it follows that

$$
\frac{\mathrm{d} \xi}{\mathrm{d} s}=1, \quad \frac{\mathrm{d} \psi}{\mathrm{d} s}=-S_{r} F^{\prime}(\phi),
$$

which, eliminating $s$, implies that the characteristic curves are given by solving

$$
\frac{\mathrm{d} \psi}{\mathrm{d} \xi}=-S_{r} F^{\prime}(\phi)=c(\phi) .
$$

Since $\phi$ is constant on each curve, the gradient of the characteristic, $c(\phi)$, is also constant. Thus, in $(\xi, \psi)$ space, the characteristics are straight lines, whose gradient is equal to the gradient of the flux function, i.e. $c(\phi)=-S_{r} F^{\prime}(\phi)$, as shown in figure $5(a, b)$. If there is a discontinuity in the concentration field at $\left(\xi_{\lambda}, \psi_{\lambda}\right)$, with $\phi_{+}$ on the forward side and $\phi_{-}$on the rearward side (defined in the direction of increasing $\psi)$, then, provided $\phi_{+}>\phi_{-}$, the gradients satisfy $c\left(\phi_{+}\right)>c\left(\phi_{-}\right)$and the characteristics diverge from one another. The void between the diverging characteristics is filled by a rarefaction fan, centred at $\left(\xi_{\lambda}, \psi_{\lambda}\right)$, within which the concentration is determined by characteristics with concentrations lying in the range $\phi \in\left[\phi_{-}, \phi_{+}\right]$, as shown in figure $5(c, d)$.

Conversely, if the initial discontinuity is such that $\phi_{+}<\phi_{-}$, then, for a convex flux function, the gradients satisfy $c\left(\phi_{+}\right)<c\left(\phi_{-}\right)$, and the characteristics intersect to form a shock. The shock path is governed by the jump condition, which can be derived from an integral form of (4.3) using a limiting argument (see e.g. Chadwick 1976; Gray \& Thornton 2005). The jump condition for (4.3) in the mapped coordinate system is

$$
-\llbracket \phi \rrbracket \frac{\mathrm{d} \psi}{\mathrm{d} \xi}+\llbracket-S_{r} F(\phi) \rrbracket=0,
$$

where the 'jump' brackets $\llbracket \cdot \rrbracket$ indicates the difference in the enclosed quantity between the forward (+) and rearward (-) sides of the shock. This implies that the gradient of the shock is

$$
\frac{\mathrm{d} \psi}{\mathrm{d} \xi}=-S_{r} \frac{\llbracket F(\phi) \rrbracket}{\llbracket \phi \rrbracket}=c\left(\phi_{+}, \phi_{-}\right),
$$

where the function $c\left(\phi_{+}, \phi_{-}\right)$, with two arguments $\phi_{+}$and $\phi_{-}$, is the gradient of the chord joining $\phi_{+}$with $\phi_{-}$on the segregation flux curve $-S_{r} F(\phi)$, as shown in figure $5(e)$. Since the characteristics on the forward $(+)$ side have a lower gradient than those on the rearward (-) side, all the characteristics intersect with the shock, as shown in figure $5(f)$ for the cubic convex flux function with $\gamma=0.35$ and $S_{r}=1.0$. 
(a)

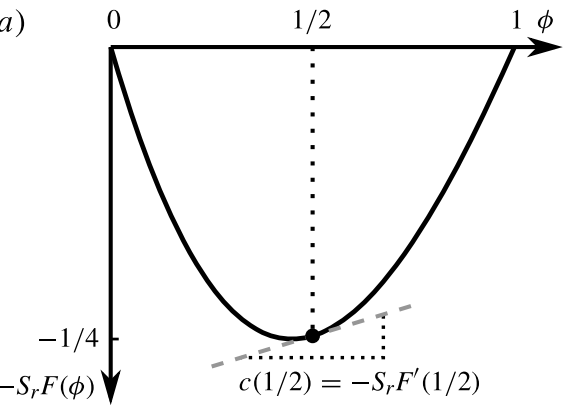

(c)

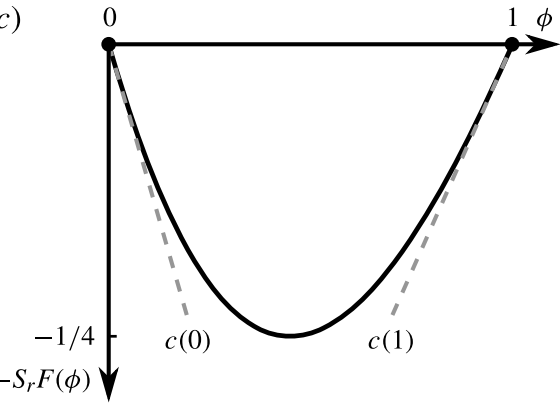

$(e)$

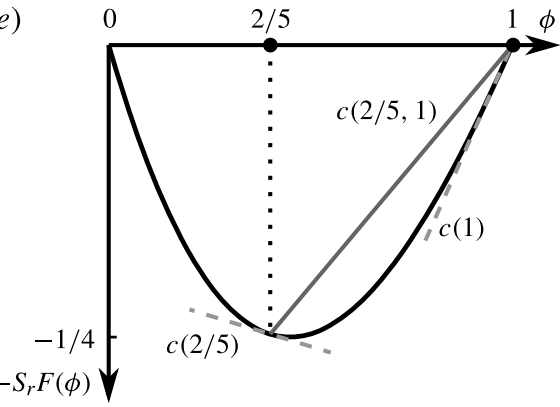

(b)

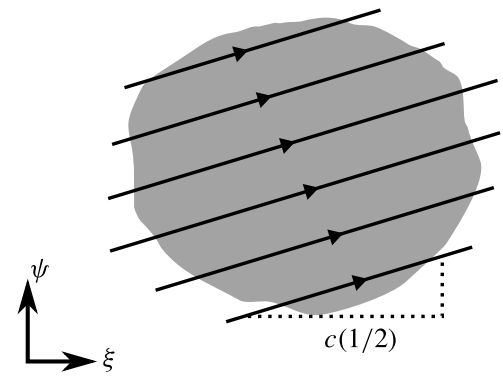

$(d)$

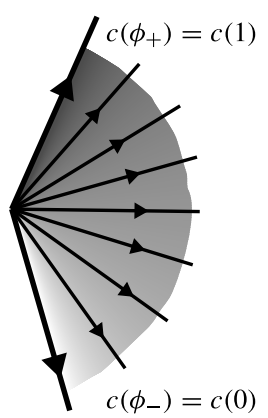

$(f)$ $c\left(\phi_{+}, \phi_{-}\right)=c(2 / 5,1)$

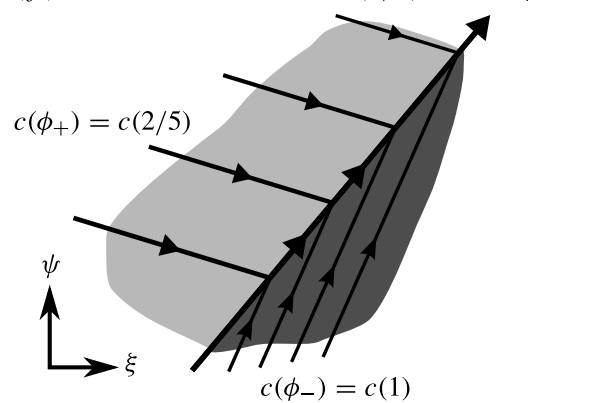

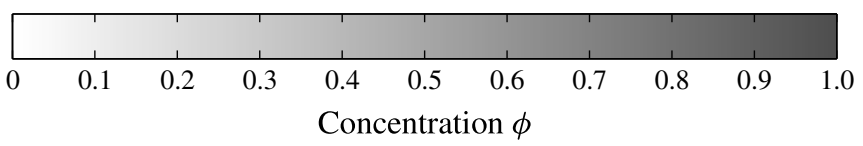

FIgURE 5. The segregation flux $-S_{r} F(\phi)$ determines the structure of the solution by setting the gradient of the characteristics $c(\phi)=-S_{r} F^{\prime}(\phi)$ (4.7), as shown for the convex function (3.4) with $\gamma=0.35$ and $S_{r}=1.0$. At concentration $\phi=1 / 2$, the gradient $c(1 / 2)$ is tangent to the flux curve $(a)$ and has the same gradient as the characteristics $(b)$. For a discontinuity $(c)$ with $\phi_{+}=1$ above and $\phi_{-}=0$ below, the gradients satisfy $c(1)>c(0)$ and so the characteristics $(d)$ diverge. The region is filled with a rarefaction fan. While for a discontinuity $(e)$ with $\phi_{+}=2 / 5$ and $\phi_{-}=1$, the gradients satisfy $c(2 / 5)<c(1)$ and the characteristics $(f)$ converge to form a shock. The gradient of the shock is set by the gradient $c(2 / 5,1)$ of the chord joining $\phi_{+}$to $\phi_{-}$on the flux curve $(e)$. 
For convex flux functions, the gradient is monotonically increasing with increasing $\phi$, so $c\left(\phi_{+}\right)<c\left(\phi_{-}\right)$when $\phi_{+}<\phi_{-}$, and the characteristics always converge. However, in the non-convex case, there is an inflection point at $\phi_{\text {inf }}$, which implies that the gradients of the characteristics increase up to $\phi_{\text {inf }}$, but decrease again afterwards. If two states $\phi_{+}<\phi_{-}$that lie on either side of the inflection point are joined by a chord with gradient $c\left(\phi_{+}, c_{-}\right)$, then the $\phi_{+}$characteristics will intersect with the shock, but the $\phi_{-}$characteristics will diverge away from it, which is unphysical. The non-convex flux functions therefore introduce a new feature into the solution, a semi-shock, which has the $\phi_{+}$characteristics intersecting it from one side, and characteristics tangential to it on the other side. In this problem, an expansion fan lies adjacent to the semi-shock with characteristics of concentrations between $\phi_{+}^{\circ}$ and $\phi_{-}$. By the definition of the image point (3.6), the $\phi_{+}^{\circ}$ characteristic lies tangential to the shock, and so characteristics only intersect with the semi-shock from one side. In general, a shock is only admissible if the area bounded by the flux curve, $-S_{r} F(\phi)$, lies completely to the left of the chord joining $\phi_{-}$to $\phi_{+}$when traversed from $\phi_{-}$ to $\phi_{+}$(Rhee et al. 1986; Laney 1998). There is a well-defined flow direction due to the bulk velocity $u(z)$, so both $x$ and $\xi$ are time-like variables. The above admissible shock condition is thus equivalent to the entropy condition of Oleinik (1959), and is compatible with the entropy condition of Lax (1957) for the convex case. Non-convex scalar conservation laws have been widely analysed in other contexts, for example in two-phase porous media flow (Buckley \& Leverett 1942), and so the semi-shock construction may also be found in the literature as a 'generalised Lax shock', a 'one-sided contact discontinuity' or an 'intermediate discontinuity' (e.g. Liu 1974; Jeffrey 1976).

For example, consider the cubic flux function (3.4) with $\gamma=0.9$ in figure 6. The area bounded by the flux curve in figure 6(a) lies to the left as one moves along the chord from $\phi_{-}=0.65$ to $\phi_{+}=0.0$, and so the lower $\phi_{-}=0.65$ characteristics collide with the upper $\phi=0.0$ characteristics to form the shock shown in figure 6(b). However, a shock between $\phi_{-}=0$ and $\phi_{+}=1$ is not admissible, because the area bounded by the flux curve lies to the right as one moves along the chord joining $\phi_{-}=0$ to $\phi_{+}=1$, in figure 6(c). A single rarefaction fan is also not possible owing to the presence of the inflection point $0<\phi_{\text {inf }}=0.704<1$. Instead, a semi-shock and an adjacent expansion fan are formed. The semi-shock joins $\phi_{+}=1$ and its image point $\phi_{-}=1^{\circ}$, whilst the expansion fan contains the characteristics generated by $\phi \in\left[0,1^{\circ}\right]$. As one now moves along the chord from $\phi_{-}=1^{\circ}$ to $\phi_{+}=1$, the dark grey area in figure $6(c)$ lies to the left and so the shock is admissible. The structure of the semi-shock and the adjacent fan is shown in figure $6(d)$. Semi-shock-fan structures also occur for $\phi_{-}=1.0$ and $\phi_{+}=\phi_{0}$, when $\phi_{0}$ lies in the range $\phi_{\mathrm{D}}<\phi_{0}<\phi_{\text {inf }}$. Finally, the area bounded by the flux function lies to the right of the chord joining $\phi_{-}=1$ to $\phi_{+}=0.75$, as shown in figure 6(e), so a shock is not admissible, and a semi-shock does not form because the inflection point $\phi_{i n f}=0.704$ does not lie in the interval $\left(\phi_{+}, \phi_{-}\right)$. Instead, a rarefaction fan forms, as shown in figure $6(f)$.

\section{Homogeneous inflow}

Following Gray \& Thornton (2005), the steady-state solution is now constructed for segregation in a steady uniform flow (Pouliquen 1999; Rognon et al. 2007; Forterre $\&$ Pouliquen 2008) in which the inflow at $\xi=0$ is of homogeneous concentration $\phi_{0}$ through the depth,

$$
\phi(0, \psi)=\phi_{0}, \quad 0 \leqslant \psi \leqslant 1 .
$$



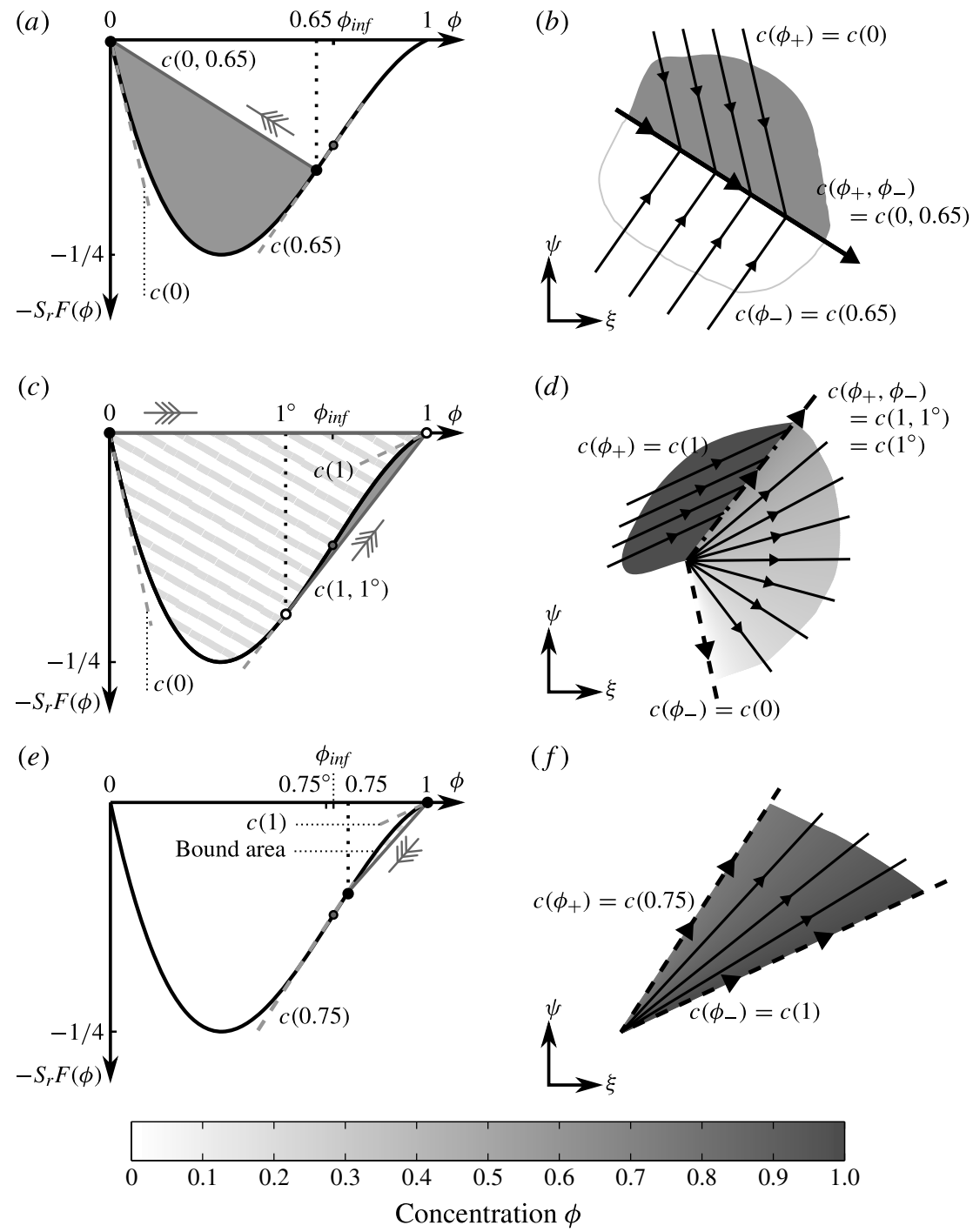

FIgURE 6. Non-convex flux functions, such as the cubic function (3.4) with $\gamma=0.9$ and $S_{r}=1$, are more complicated because of the inflection point $\phi_{i n f}$, which causes the maximum large-particle velocity to occur at $\phi=\phi_{\text {crit }}=\phi_{\mathrm{C}}$. In order for a shock to be admissible, the area bounded by the flux curve must lie to the left of the chord joining $\phi_{-}$to $\phi_{+}$. (a) When $\phi_{-}=0.65$ and $\phi_{+}=0$, the bounded area (grey) lies completely to the left as one moves along the chord from $\phi_{-}$to $\phi_{+}$in the direction indicated, so $(b)$ the shock is admissible and the characteristics converge from either side. (c) The cross-hatched area lies to the right of the chord joining $\phi_{-}=0$ and $\phi_{+}=1$, and so the shock is not admissible. However, the area (grey) lies to the left of the chord joining $\phi=1^{\circ}$ to $\phi=1$. A semi-shock $(d)$ is therefore formed, whose gradient is equal to the gradient of the $\phi=1^{\circ}$ characteristic, together with an adjacent fan for $\phi \in\left[0,1^{\circ}\right]$. The semi-shock coincides with the $\phi=1.0^{\circ}$ characteristic that is at the edge of the rarefaction fan. (e) The cross-hatched and labelled area lies to the right of the chord joining $\phi_{-}=1$ to $\phi_{+}=0.75$, and so a shock is not admissible. In addition, a semi-shock does not form because the inflection point $\phi_{\text {inf }}$ does not lie in the range $[0.75,1] .(f)$ Instead, the characteristics diverge to form a rarefaction fan. 

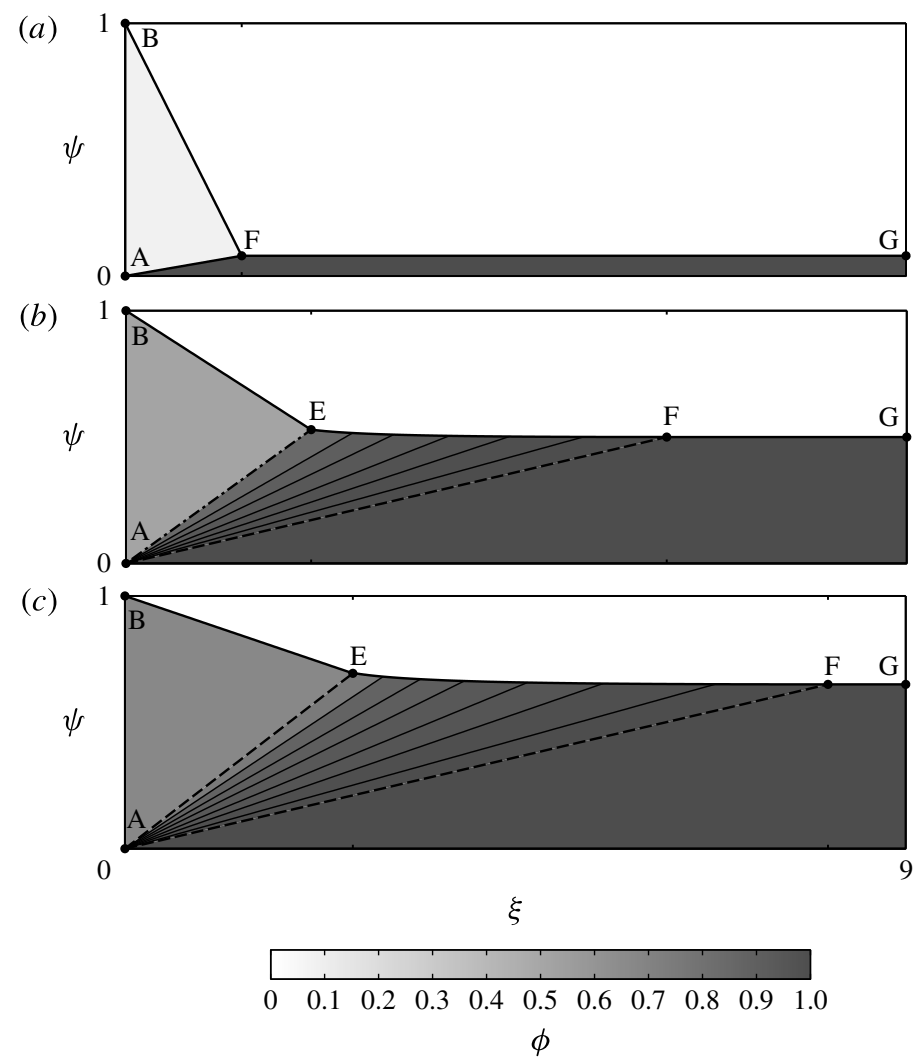

FIGURE 7. Exact solutions for the small-particle concentration $\phi$ in mapped coordinates $(\xi, \psi)$ using the cubic flux function (3.4) with $\gamma=0.9$ and $S_{r}=0.5$. At the inflow $(\xi=0)$, the grains are initially homogeneously mixed with concentration $(a) \phi_{0}=0.08$, (b) $\phi_{0}=0.50$ and (c) $\phi_{0}=0.65$. Thick solid lines represent shocks, thick dash-dotted lines represent semi-shocks whilst thick dashed lines represent the edge of rarefaction fans. Characteristics are shown with thin solid black lines.

There are three distinct solution structures that are dependent on $\phi_{0}$ and the particular form of the flux function $F(\phi)$. For convex flux functions, and non-convex flux functions with the condition $\phi_{0} \leqslant \phi_{\mathrm{D}}$, where $\phi_{\mathrm{D}}$ is defined in (3.9), the solution consists of three shocks separating the homogeneous inflow from a layer of large particles above and a layer of small particles below. Concentration $\phi_{\mathrm{D}}$ is significant because it is the maximum concentration at which the image point $\phi^{\circ}$ lies outside the range $[0,1)$. These solutions therefore have essentially the same structure as those for the quadratic flux (Gray \& Thornton 2005), as shown in figure 7(a). Non-convex flux functions also give rise to two new solutions. When the initial concentration is in the range $\phi_{\mathrm{D}}<\phi_{0}<\phi_{\text {inf }}$, a semi-shock with an adjacent fan separates the homogeneous region from the lower layer of small particles, as shown in figure $7(b)$. If the initial concentration is increased further, $\phi_{0}>\phi_{i n f}$, the homogeneous region and region of small particles are separated by just a rarefaction fan, as in figure $7(c)$.

These new structures arise because, for $\phi>\phi_{\text {crit }}$, the velocity of large particles decreases with increasing small-particle concentration. A few large particles therefore rise at a very slow rate, and so they are swept a long distance downstream with the 


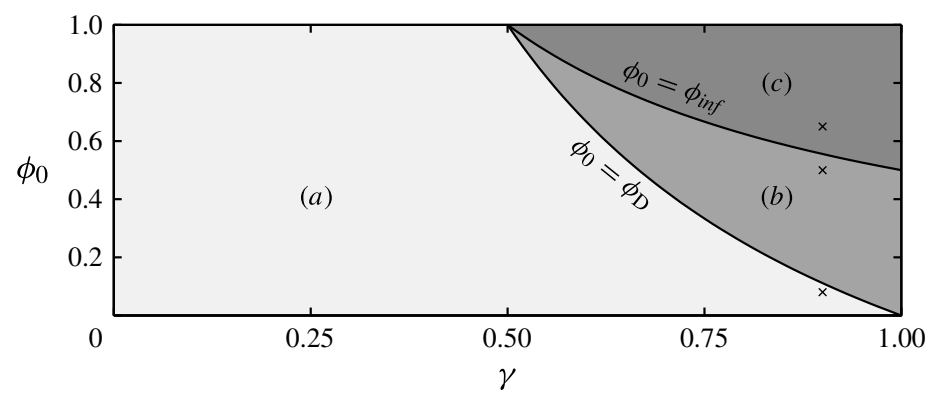

FIGURE 8 . The $\left(\gamma, \phi_{0}\right)$ parameter space for the homogeneous inflow problem (figure 7) using the cubic family of flux functions (3.4). (a) A three-shock solution similar to that in Gray \& Thornton (2005) occurs for convex flux functions $(\gamma \leqslant 0.5)$ and non-convex functions $(\gamma>0.5)$ with a low inflow concentration of small particles, $\phi_{0}<\phi_{\mathrm{D}}$. $(b)$ For non-convex flux functions $\gamma>0.5$ with an inflow concentration of small particles $\phi_{\mathrm{D}} \leqslant$ $\phi_{0}<\phi_{\text {inf }}$, a semi-shock with an adjacent fan separates the homogeneous region from the region of small particles. (c) For even higher initial concentrations of small particles, $\phi_{0} \geqslant$ $\phi_{\text {inf }}$, this separation becomes a smooth rarefaction fan. The curves $\phi_{0}=\phi_{\mathrm{D}}$ and $\phi_{0}=\phi_{\text {inf }}$ are given by (3.9) and (3.5) respectively. Parameter values for the structures in figure 7 are marked with crosses.

bulk flow before they finally join the large-particle layer above. All three solutions are found using the family of cubic flux functions (3.4) parametrised by $\gamma$, with $F(\phi)$ convex for $\gamma \leqslant 0.5$ and non-convex for $0.5<\gamma \leqslant 1$. The $\left(\gamma, \phi_{0}\right)$ parameter space is shown in figure 8 , with regions $(a-c)$ corresponding to the three different structures in figure $7(a-c)$, respectively. The three structures in $(\xi, \psi)$ space are explained in more detail below, and can be transformed back to physical $(x, z)$ space using the results of $\S 6.4$, as shown in, for example, Gray \& Thornton (2005), Thornton et al. (2006), Thornton \& Gray (2008) and Gray \& Ancey (2011). Note that this inverse transformation locally stretches and compresses the normal coordinate, but leaves the downstream coordinate unaffected.

\subsection{Three-shock solution}

The characteristics sweep the initial concentration downstream to create a region adjacent to the inflow that is still at $\phi=\phi_{0}$. Within this region, there is a flux of small particles percolating downwards, and an equal and opposite flux of large grains being squeezed upwards. However, at the base, $\psi=0$, the boundary condition (2.21) implies that there is no flux of large particles and, consequently, the small particles have to separate out into a pure phase across the concentration shock AF shown in figure 7(a). This can be computed by solving the jump condition (4.9) with $\phi_{+}=\phi_{0}$ and $\phi_{-}=1$, subject to the condition that the shock starts at point $\mathrm{A}$, which has coordinates $(0,0)$, to give the straight line

$$
\psi_{\mathrm{AF}}=S_{r} \frac{F\left(\phi_{0}\right)}{1-\phi_{0}} \xi
$$

Similarly, the upper shock BF separates the homogeneous region $\left(\phi_{-}=\phi_{0}\right)$ from the layer of large particles $\left(\phi_{+}=0\right)$ that collect at the surface. By integrating (4.9), subject 
to the initial condition that the shock starts from point B with coordinates $(0,1)$, shock $\mathrm{BF}$ has coordinates $\left(\xi, \psi_{\mathrm{BF}}\right)$ given by

$$
\psi_{\mathrm{BF}}=1-S_{r} \frac{F\left(\phi_{0}\right)}{\phi_{0}} \xi
$$

The two shocks $\mathrm{AF}$ and $\mathrm{BF}$ meet at point $\mathrm{F}$, whose coordinates $\left(\xi_{\mathrm{F}}, \psi_{\mathrm{F}}\right)$ are found by equating (5.2) and (5.3) to give

$$
\xi_{\mathrm{F}}=\frac{\phi_{0}\left(1-\phi_{0}\right)}{S_{r} F\left(\phi_{0}\right)}, \quad \psi_{\mathrm{F}}=\phi_{0} .
$$

At point $\mathrm{F}$ the flow has reached its final segregated state with a third shock FG separating the two inversely graded layers, with large particles above $\left(\phi_{+}=0\right)$ and small particles below $\left(\phi_{-}=1\right)$. Integrating (4.9) implies that the shock FG has coordinates

$$
\psi_{\mathrm{FG}}=\phi_{0}, \quad \xi \geqslant \xi_{\mathrm{F}},
$$

which is independent of $F$ (and hence $\gamma$ ), as the flux function and the asymmetry have no influence on the final non-diffuse segregated state. Convex flux functions always give rise to this three-shock structure, and hence the solution resembles that derived by Gray \& Thornton (2005) for the quadratic flux function.

\subsection{Semi-shock solution with adjacent fan}

For non-convex flux functions with an inflow concentration $\phi_{\mathrm{D}} \leqslant \phi_{0} \leqslant \phi_{\text {inf }}$, the homogeneous region and the lower layer of small particles are separated by a semi-shock AE with an adjacent expansion fan AEFA centred at point $\mathrm{A}=(0,0)$, as shown in figure $7(b)$. Semi-shock AE starts at point $\mathrm{A}$ and separates the characteristics of the homogeneous region $\phi_{+}=\phi_{0}$ from the $\phi_{-}=\phi_{0}^{\circ}$ characteristic, which lies immediately adjacent and tangential to the shock. From (3.6) and (4.9), it follows that the semi-shock $\mathrm{AE}$ has coordinates $\left(\xi, \psi_{\mathrm{AE}}\right)$ given by

$$
\psi_{\mathrm{AE}}=-S_{r} F^{\prime}\left(\phi_{0}^{\circ}\right) \xi
$$

with $0 \leqslant \xi \leqslant \xi_{\mathrm{E}}$. The rarefaction fan occurs between the $\phi=1$ and $\phi_{0}^{\circ}$ characteristics, and is given by

$$
\psi=-S_{r} F^{\prime}(\phi) \xi, \quad \text { for } \phi \in\left[\phi_{0}^{\circ}, 1\right] .
$$

The upper shock BE separates the homogeneous region from the layer of large particles above and satisfies the same equation as shock BF in the three-shock structure (5.3). The semi-shock AE meets the upper shock BE at point $\mathrm{E}$, which from (5.3) and (5.6) has coordinates

$$
\xi_{\mathrm{E}}=\frac{\phi_{0}}{S_{r}\left(F\left(\phi_{0}\right)-\phi_{0} F^{\prime}\left(\phi_{0}^{\circ}\right)\right)}, \quad \psi_{\mathrm{E}}=-S_{r} F^{\prime}\left(\phi_{0}^{\circ}\right) \xi_{\mathrm{E}} .
$$

Another shock EF separates the rarefaction fan $\left(\phi_{-}=\phi\right)$ from the layer of large particles above $\left(\phi_{+}=0\right)$. Using the chain rule, the shock gradient (4.9) can be written as

$$
\frac{\mathrm{d} \psi}{\mathrm{d} \phi}=-S_{r} \frac{F(\phi)}{\phi} \frac{\mathrm{d} \xi}{\mathrm{d} \phi}
$$


while the equation for the characteristics of the fan (5.7) can be differentiated with respect to $\phi$ to give

$$
\frac{\mathrm{d} \psi}{\mathrm{d} \phi}=-S_{r} F^{\prime \prime}(\phi) \xi-S_{r} F^{\prime}(\phi) \frac{\mathrm{d} \xi}{\mathrm{d} \phi} .
$$

Equating (5.9) and (5.10) yields an ordinary differential equation (ODE) for the shock path $\mathrm{EF}$,

$$
\frac{1}{\xi} \frac{\mathrm{d} \xi}{\mathrm{d} \phi}=\frac{\phi F^{\prime \prime}(\phi)}{F(\phi)-\phi F^{\prime}(\phi)},
$$

which can be integrated, subject to the initial condition that the shock starts from point $\left(\xi_{\mathrm{E}}, \psi_{\mathrm{E}}\right)$. Using (5.7) and the definition of the image point (3.6) to reformulate the initial condition (5.8), it follows that the shock EF is parametrised by

$$
\xi_{\mathrm{EF}}=\frac{\phi_{0}}{S_{r}\left(F(\phi)-\phi F^{\prime}(\phi)\right)}, \quad \psi_{\mathrm{EF}}=-S_{r} F^{\prime}(\phi) \xi_{\mathrm{EF}}, \quad \text { for } \phi \in\left[\phi_{0}^{\circ}, 1\right] .
$$

Shock EF ends at $\mathrm{F}$, whose coordinates are given by (5.12) with $\phi=1$, i.e.

$$
\xi_{\mathrm{F}}=\frac{\phi_{0}}{-S_{r} F^{\prime}(1)}, \quad \psi_{\mathrm{F}}=\phi_{0} .
$$

A final shock FG separates the large- and small-particle layers in the final inversely graded state, as in (5.5).

\subsection{Rarefaction fan solution}

Here the solution is essentially the same as that in $\S 5.2$ except that there is no semishock, i.e. the homogeneous inflow and the layer of small particles are separated by a rarefaction fan AEFA centred at point $\mathrm{A}=(0,0)$. The characteristics in the expansion fan (5.7) now lie in the range $\phi \in\left[\phi_{0}, 1\right]$. The left-hand edge of the rarefaction fan $\mathrm{AE}$ is given by the $\phi=\phi_{0}$ characteristic, and therefore $\phi_{0}$ replaces $\phi_{0}^{\circ}$ in (5.8) for $\mathrm{E}$. Similarly, shock EF given by (5.12) is now parametrised by $\phi \in\left[\phi_{0}, 1\right]$.

\subsection{Final segregation distance}

For the class of asymmetric flux functions $F(\phi)$ under consideration, if $F(\phi)$ is convex, or $F(\phi)$ is non-convex and $\phi_{0} \leqslant \phi_{\mathrm{D}}$, then the maximum segregation distance $\xi_{\mathrm{F}}$ is given by (5.4), while if $F(\phi)$ is non-convex and $\phi_{0}>\phi_{\mathrm{D}}$, the maximum segregation distance is given by (5.13). For the cubic family of flux functions (3.4), this implies that

$$
\xi_{\mathrm{F}}=\left\{\begin{array}{lll}
\frac{1}{S_{r} A_{\gamma}\left(1-\gamma \phi_{0}\right)}, & \gamma \leqslant 0.5 \quad \text { or } \gamma>0.5 \quad \text { with } \phi_{0} \leqslant \phi_{\mathrm{D}}, \\
\frac{\phi_{0}}{S_{r} A_{\gamma}(1-\gamma)}, & \gamma>0.5 \quad \text { with } \phi_{0}>\phi_{\mathrm{D}},
\end{array}\right.
$$

which is an increasing function of $\phi_{0}$, as shown in figure 9 . Note that, for the quadratic model (2.14), when $\gamma=0$, the segregation distance $\xi_{\mathrm{F}}=1 / S_{r}$, which is independent of the inflow concentration, as shown by the grey line in figure 9 . The dashed line shows the segregation distance for the cubic flux function with $\gamma=0.35$. There is a weak dependence on $\phi_{0}$ : for $\phi_{0}<1 / 2$, the distance for complete segregation $\xi_{\mathrm{F}}$ is shorter than in the quadratic model, reflecting the fact that there 


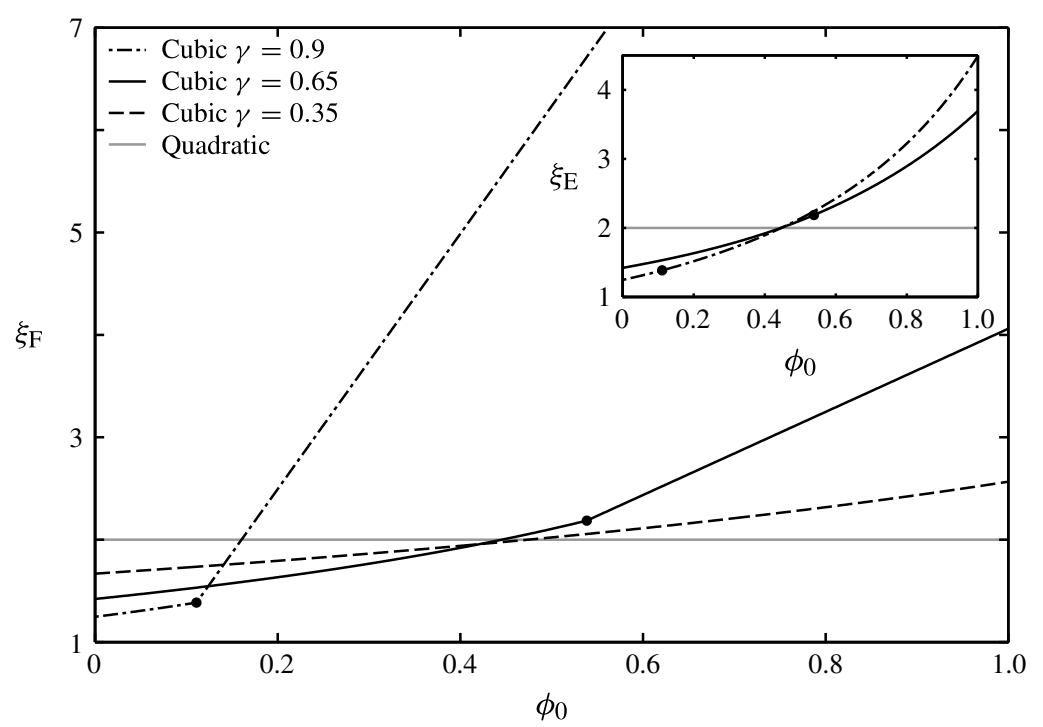

FIGURE 9. The maximum segregation distance $\xi_{\mathrm{F}}$ plotted as a function of the inflow concentration $\phi_{0}$ for the quadratic model (grey line), the convex cubic model with $\gamma=0.35$ (dashed line) and two cases of the non-convex cubic model (solid and dot-dashed lines) for $\gamma=0.65$ and 0.9 , respectively. The black circles denote the change in the functional form of $\xi_{\mathrm{F}}$ in (5.14) due to the presence of a rarefaction fan in the solution structure. The curves have been plotted for $S_{r}=0.5$. Inset: The intermediate point $\xi_{\mathrm{E}}$ given by (5.8) also increases with the inflow concentration $\phi_{0}$, showing that the increasing behaviour of the final segregation distance should be identifiable in experiments.

are only a few small particles that percolate down faster; whilst for $\phi_{0}>1 / 2$, the segregation distance is longer than in the quadratic model, reflecting the fact that a few large particles segregate to the top at a slower rate, when they are surrounded by high concentrations of fines, as shown in figure 4. This effect becomes stronger with increasing $\gamma$. The non-convex models with $\gamma=0.65$ and $\gamma=0.9$ (solid and dot-dashed lines, respectively) exhibit the same behaviour, as well as a change in the dependence of $\xi_{\mathrm{F}}$ at $\phi_{\mathrm{D}}$, which are shown by the black circles in figure 9. This is the point where the lower shock becomes a semi-shock with an adjacent expansion fan. At $\phi_{\mathrm{D}}$, the segregation distance $\xi_{\mathrm{F}}$ switches from the first to the second formula in (5.14), which has a much stronger dependence on the inflow concentration $\phi_{0}$ owing to the decreasing rise rate of the large particles at high concentrations of fines. Moreover, in the limit as $\gamma \rightarrow 1$, the rarefaction fan becomes infinitely wide, because there is a double zero in the flux function (3.4) and the large-particle rise rate tends to zero at the outer edge of the expansion fan, $w_{l}(1) \rightarrow 0$.

As the concentration difference between $\mathrm{E}$ and $\mathrm{F}$ is small, small amounts of diffusive remixing may cause the two points to be indistinguishable in experiments. However, the inset of figure 9 shows that the intermediate distance $\xi_{\mathrm{E}}$ given by (5.8) also shows increasing behaviour with $\phi_{0}$, and so an experimental measure of the final segregation distance is predicted to show increasing behaviour.

\section{Normally graded inflow}

Consider next the normally graded (see e.g. Tucker 2003) inflow problem of Thornton et al. (2006), where the small particles initially lie on top of the large 


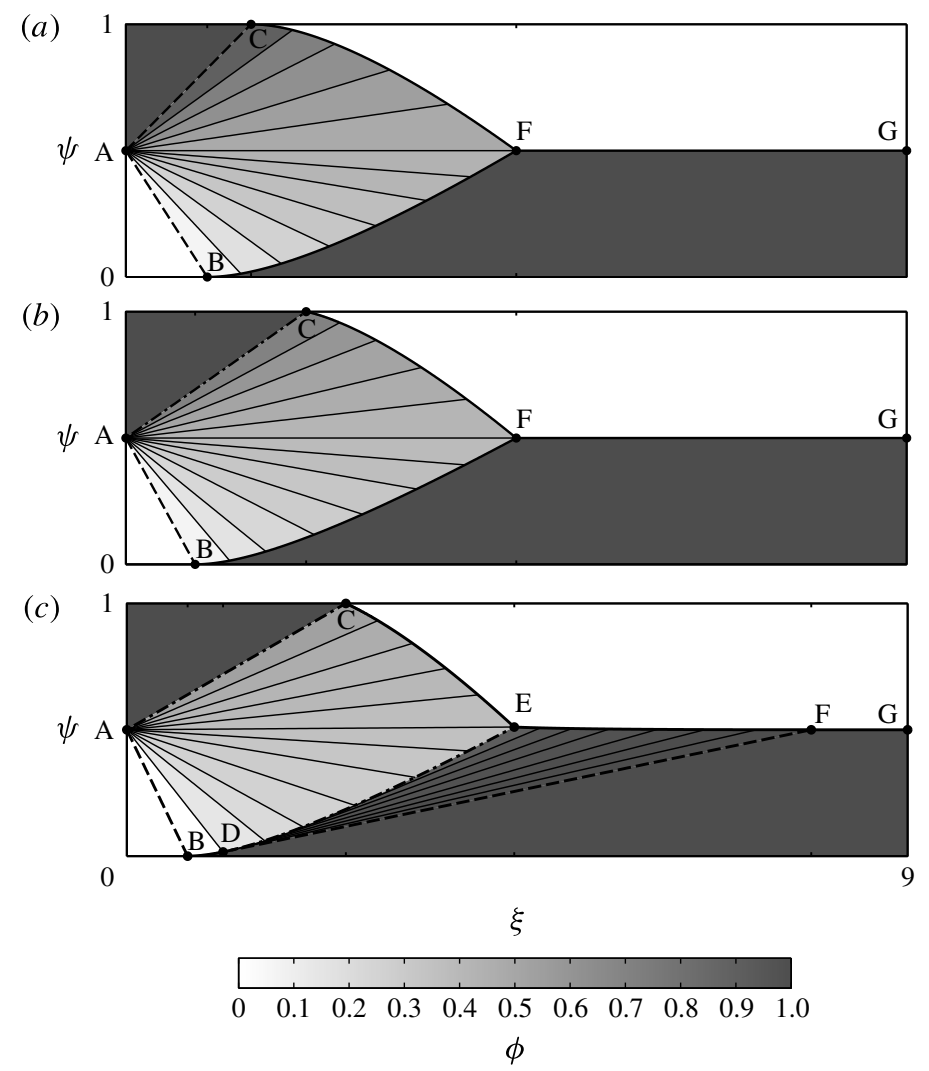

FIGURE 10. Exact solutions for an initially normally graded mixture at the inflow $\xi=0$, where $\psi_{\mathrm{A}}=1 / 2$ and $S_{r}=0.5$. The cubic flux function (3.4) has been used with $(a) \gamma=$ $0.35,(b) \gamma=0.65$ and $(c) \gamma=0.90$. Thick solid lines represent shocks, thick dot-dashed lines are semi-shocks, whilst thick dashed lines represent the edge of rarefaction fans. Characteristics are shown with thin solid black lines.

particles. The inflow at $\xi=0$ thus satisfies

$$
\phi(0, \psi)= \begin{cases}1, & \psi_{\mathrm{A}} \leqslant \psi \leqslant 1, \\ 0, & 0 \leqslant \psi<\psi_{\mathrm{A}},\end{cases}
$$

where $\psi_{\mathrm{A}}$ is the height of the initial discontinuity. The small particles, which are initially at the top, percolate downwards and accumulate at the bottom, whilst the large particles at the bottom are squeezed upwards and accumulate at the top. This causes a transition from the initial normally graded concentration profile to a final inversely graded concentration profile some distance downstream.

There are three distinct solution structures for the concentration field that are dependent on the initial discontinuity height $\psi_{\mathrm{A}}$ and the nature of the flux function $F(\phi)$. These are shown for the cubic flux function (3.4) in figure 10. Convex flux functions $(\gamma=0.35)$ give the simple structure shown in figure 10(a). A rarefaction fan develops, which separates the small particles above from the large particles below. The leading-edge characteristics of the fan, $\phi=0$ and $\phi=1$, intersect the base and the free surface at B and C, respectively, and two shocks are generated that eventually intersect 


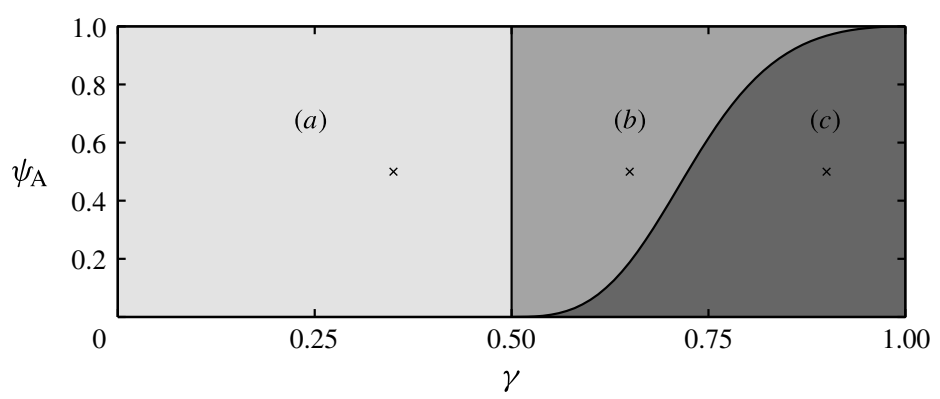

FIgURE 11. The $\left(\gamma, \psi_{\mathrm{A}}\right)$ parameter space for the three distinct structures $(a-c)$ shown in figure 10. There is one solution for $(a)$ convex flux functions $(\gamma \leqslant 0.5)$, whilst for non-convex flux functions $(\gamma>0.5)$ either $(b)$ or $(c)$ may occur. The curve separating these two states is given by (6.26). Increasing the cubic asymmetry parameter $\gamma$, or decreasing the initial discontinuity height $\psi_{\mathrm{A}}$, causes the transition from structure $(b)$ to $(c)$. The parameter values used in both figures 10 and 13 are marked with crosses.

to create the final reverse-graded state. This is similar to the solution structure for the quadratic flux derived by Thornton et al. (2006). One key difference is that, for the quadratic flux, points $\mathrm{B}$ and $\mathrm{C}$ lie the same distance downstream for $\psi_{\mathrm{A}}=1 / 2$, but for the asymmetric flux functions they do not. Non-convex flux functions $(\gamma=0.65,0.9)$ give rise to two new structures shown in figure $10(b, c)$. The initial pure phase layers are separated by a semi-shock between the rarefaction fan and the initial upper layer of small particles. The expansion fan is always separated from the final upper pure phase of large particles by a shock; however, it may be separated from the lower layer of small particles by either a single shock (figure 10b) or a semi-shock with another adjacent fan (figure 10c), leading to two different structures. When the cubic flux function is used, the three structures have the $\left(\gamma, \psi_{\mathrm{A}}\right)$ parameter dependence shown in figure 11, where regions $(a-c)$ correspond to the three different structures in figure $10(a-c)$, respectively. As the cubic asymmetry parameter $\gamma$ is increased or the initial discontinuity height $\psi_{\mathrm{A}}$ is decreased, the structure changes from figure $10(b)$ to $(c)$. The three solution structures will be examined in more detail below. It is also shown how to transform the solutions in velocity-integrated coordinates $(\xi, \psi)$, given by equation (4.2), back to physical $(x, z)$ space.

\subsection{Structure for convex flux functions}

The simple structure in figure 10(a) occurs for convex flux functions, such as the cubic flux function (3.4) with $\gamma \leqslant 0.5$. The initial inversely graded layers are separated by a rarefaction fan centred at point $\mathrm{A}$, whose characteristics are given by

$$
\psi=\psi_{\mathrm{A}}-S_{r} F^{\prime}(\phi) \xi, \quad \text { for } \phi \in[0,1] \text {. }
$$

The $\phi=1$ characteristic determines point $\mathrm{C}$, where the first large particle reaches the surface, whilst the $\phi=0$ characteristic determines point $\mathrm{B}$, where the first small particle reaches the base. Points $\mathrm{B}$ and $\mathrm{C}$ have coordinates $\left(\xi_{\mathrm{B}}, 0\right)$ and $\left(\xi_{\mathrm{C}}, 1\right)$ respectively, where

$$
\begin{aligned}
& \xi_{\mathrm{B}}=\frac{\psi_{\mathrm{A}}}{S_{r} F^{\prime}(0)}=\frac{\psi_{\mathrm{A}}}{S_{r} A_{\gamma}}, \\
& \xi_{\mathrm{C}}=-\frac{1-\psi_{\mathrm{A}}}{S_{r} F^{\prime}(1)}=\frac{1-\psi_{\mathrm{A}}}{S_{r} A_{\gamma}(1-\gamma)},
\end{aligned}
$$


with the general result followed by the result specific to the cubic flux function (3.4). A shock $\mathrm{CF}$ with coordinates $\left(\psi_{\mathrm{CF}}, \xi_{\mathrm{CF}}\right)$ starts from point $\mathrm{C}$ and separates the rarefaction fan $\left(\phi_{-}=\phi\right)$ from the layer of large particles that accumulates at the top $\left(\phi_{+}=0\right)$. In the same manner as the derivation of (5.11), the equation for the rarefaction characteristics (6.2) may be differentiated and combined with the shock gradient (4.9) to give an implicit equation governing shock CF,

$$
\frac{1}{\xi} \frac{\mathrm{d} \xi}{\mathrm{d} \phi}=\frac{\phi F^{\prime \prime}(\phi)}{F(\phi)-\phi F^{\prime}(\phi)} .
$$

This can be integrated with the initial condition that the shock starts from point $\mathrm{C}$ to give coordinates $\left(\xi_{\mathrm{CF}}, \psi_{\mathrm{CF}}\right)$ as

$$
\xi_{\mathrm{CF}}=\frac{1-\psi_{\mathrm{A}}}{S_{r}\left(F(\phi)-\phi F^{\prime}(\phi)\right)}, \quad \psi_{\mathrm{CF}}=\psi_{\mathrm{A}}-S_{r} F^{\prime}(\phi) \xi_{\mathrm{CF}}, \quad \text { for } \phi \in\left[\phi_{\mathrm{F}}, 1\right], \quad(6.6 a, b)
$$

where the characteristic $\phi=\phi_{\mathrm{F}}$ in the expansion fan intersects with point F. Similarly, a shock BF separates the rarefaction fan $\left(\phi_{+}=\phi\right)$ from the lower layer of small particles $\left(\phi_{-}=1\right)$ with the implicit equation

$$
\frac{1}{\xi} \frac{\mathrm{d} \xi}{\mathrm{d} \phi}=\frac{-(1-\phi) F^{\prime \prime}(\phi)}{F(\phi)+(1-\phi) F^{\prime}(\phi)} .
$$

The coordinates $\left(\xi_{\mathrm{BF}}, \psi_{\mathrm{BF}}\right)$ of $\mathrm{BF}$ are found by integrating (6.7) subject to the initial condition that shock $\mathrm{BF}$ starts from point $\mathrm{B}$, yielding

$$
\xi_{\mathrm{BF}}=\frac{\psi_{\mathrm{A}}}{S_{r}\left(F(\phi)+(1-\phi) F^{\prime}(\phi)\right)}, \quad \psi_{\mathrm{BF}}=\psi_{\mathrm{A}}-S_{r} F^{\prime}(\phi) \xi_{\mathrm{BF}}, \quad \text { for } \phi \in\left[0, \phi_{\mathrm{F}}\right] . \quad(6.8 a, b)
$$

The two shocks and the $\phi=\phi_{\mathrm{F}}$ characteristic meet at point F. An equation for the concentration $\phi_{\mathrm{F}}$ is obtained by equating (6.6) to (6.8), to give

$$
\frac{1-\psi_{\mathrm{A}}}{\psi_{\mathrm{A}}}=\frac{F\left(\phi_{\mathrm{F}}\right)-\phi_{\mathrm{F}} F^{\prime}\left(\phi_{\mathrm{F}}\right)}{F\left(\phi_{\mathrm{F}}\right)+\left(1-\phi_{\mathrm{F}}\right) F^{\prime}\left(\phi_{\mathrm{F}}\right)}=\frac{\phi_{\mathrm{F}}^{2}\left(1+\gamma-2 \gamma \phi_{\mathrm{F}}\right)}{\left(1-\phi_{\mathrm{F}}\right)^{2}\left(1-2 \gamma \phi_{\mathrm{F}}\right)},
$$

where the last result is specific to the cubic flux function (3.4). The two shocks therefore meet at $\left(\xi_{\mathrm{F}}, \psi_{\mathrm{F}}\right)$, which has coordinates

$$
\xi_{\mathrm{F}}=\frac{1-\psi_{\mathrm{A}}}{S_{r}\left(F\left(\phi_{\mathrm{F}}\right)-\phi_{\mathrm{F}} F^{\prime}\left(\phi_{\mathrm{F}}\right)\right)}, \quad \psi_{\mathrm{F}}=1-\psi_{\mathrm{A}} .
$$

A third shock FG starts from point $\mathrm{F}$ separating the two inversely graded layers, with coordinates $\left(\xi, \psi_{\mathrm{FG}}\right)$,

$$
\psi_{\mathrm{FG}}=1-\psi_{\mathrm{A}}, \quad \xi \geqslant \xi_{\mathrm{F}} .
$$

Similar to (5.5), the final segregated state is independent of $F$ (and hence $\gamma$ ).

\subsection{Structure for non-convex flux functions}

The two different solution structures shown in figure $10(b, c)$ occur for non-convex flux functions, such as the cubic flux function (3.4) with $\gamma>0.5$. Within the expansion 
fan, small particles percolate downwards and large particles are squeezed upwards, as before. However, because of the non-convexity, the maximum large-particle rise rate occurs at concentration $\phi=\phi_{\text {crit }}=\phi_{\mathrm{C}}$ rather than at $\phi=1$. An expansion over the interval $\phi \in[0,1]$ (as in the convex case) is not possible, because, for $\phi$ greater than $\phi_{i n f}$, the characteristics overlap with those generated by lower values of $\phi$. Instead, a semi-shock AC is formed between the initial upper layer of small particles $\phi_{+}=1$ and the $\phi_{-}=1^{\circ}=\phi_{\mathrm{C}}$ characteristic at the edge of the fan. Integrating the shock gradient (4.9) and using the definition of the image point (3.6) implies that the coordinates $\left(\xi, \psi_{\mathrm{AC}}\right)$ of semi-shock $\mathrm{AC}$ are given by

$$
\psi_{\mathrm{AC}}=\psi_{\mathrm{A}}-S_{r} F^{\prime}\left(\phi_{\mathrm{C}}\right) \xi .
$$

The characteristics of the fan are given by (6.2) with $\phi \in\left[0, \phi_{\mathrm{C}}\right]$. The $\phi=0$ characteristic again determines point $\mathrm{B}$ for the first small particle to reach the base, which has coordinates $\left(\xi_{\mathrm{B}}, 0\right)$ given by (6.3). However, semi-shock AC now governs the position of point $\mathrm{C}$ for the first large particle to reach the surface, with coordinates $\left(\xi_{\mathrm{C}}, 1\right)$, where

$$
\xi_{\mathrm{C}}=-\frac{1-\psi_{\mathrm{A}}}{S_{r} F^{\prime}\left(\phi_{\mathrm{C}}\right)}=4 \gamma \frac{1-\psi_{\mathrm{A}}}{S_{r} A_{\gamma}} .
$$

The two different non-convex solutions are dependent on whether the concentration $\phi=\phi_{\mathrm{F}}$, defined in (6.9), satisfies $\phi_{\mathrm{F}} \leqslant \phi_{\mathrm{D}}$ or $\phi_{\mathrm{F}}>\phi_{\mathrm{D}}$. In the former case (shown in figure 10b), the rarefaction fan $\left(\phi_{-}=\phi\right)$ is separated from the upper layer of large particles $\left(\phi_{+}=0\right)$ by a shock CF. The shock starts from $\mathrm{C}$, and its coordinates $\left(\xi_{\mathrm{CF}}, \psi_{\mathrm{CF}}\right)$ are given by (6.6) with $\phi \in\left[\phi_{\mathrm{F}}, \phi_{\mathrm{C}}\right]$. A second shock BF also starts from $\mathrm{B}$ and separates the rarefaction fan $\left(\phi_{+}=\phi\right)$ from the lower layer of small particles $\left(\phi_{-}=1\right)$. Similarly, this has coordinates $\left(\xi_{\mathrm{BF}}, \psi_{\mathrm{BF}}\right)$ given by (6.8) with $\phi \in\left[0, \phi_{\mathrm{F}}\right]$. A final shock FG separates the inversely graded state, with coordinates $\left(\xi_{\mathrm{FG}}, \psi_{\mathrm{FG}}\right)$ given by (6.11).

The solution in the latter case $\left(\phi_{\mathrm{F}}>\phi_{\mathrm{D}}\right)$ is more complex and gives rise to the structure shown in figure $10(c)$. The rarefaction fan $\left(\phi_{-}=\phi\right)$ is separated from the upper layer of large particles $\left(\phi_{+}=0\right)$ by a shock $\mathrm{CE}$ that starts from point $\mathrm{C}$. The shock gradient (4.9) and the rarefaction characteristics (6.2) can be used to show that $\mathrm{CE}$ has coordinates $\left(\xi_{\mathrm{CE}}, \psi_{\mathrm{CE}}\right)$ that satisfy (6.6). This is the same equation as satisfied by the coordinates of shock CF in the non-convex case, but here $\phi$ lies in the range $\phi \in\left[\phi_{\mathrm{E}}, \phi_{\mathrm{C}}\right]$. A shock BD together with a semi-shock DE and adjacent fan DEFD separate the initial rarefaction fan $\left(\phi_{+}=\phi\right)$ from the layer of small particles that accumulates at the bottom of the flow $\left(\phi_{-}=1\right)$. Shock BD starts from $\mathrm{B}$, and integrating the shock gradient (4.9) shows that $\mathrm{BD}$ has coordinates $\left(\xi_{\mathrm{BD}}, \psi_{\mathrm{BD}}\right)$ that satisfy (6.8). This is the same equation as the coordinates of shock BF in the nonconvex case, but with $\phi \in\left[0, \phi_{\mathrm{D}}\right]$. Point $\mathrm{D}$ has coordinates $\left(\xi_{\mathrm{D}}, \psi_{\mathrm{D}}\right)$ given by (6.8) with $\phi=\phi_{\mathrm{D}}$, where

$$
\left.\begin{array}{l}
\xi_{\mathrm{D}}=\frac{\psi_{\mathrm{A}}}{S_{r}\left(F\left(\phi_{\mathrm{D}}\right)+\left(1-\phi_{\mathrm{D}}\right) F^{\prime}\left(\phi_{\mathrm{D}}\right)\right)}=\frac{\psi_{\mathrm{A}} \gamma^{2}}{S_{r} A_{\gamma}(2 \gamma-1)^{3}}, \\
\psi_{\mathrm{D}}=\psi_{\mathrm{A}}-S_{r} F^{\prime}\left(\phi_{\mathrm{D}}\right) \xi_{\mathrm{D}}=\psi_{\mathrm{A}} \frac{(1-\gamma)^{2}(3 \gamma-1)}{(2 \gamma-1)^{3}} .
\end{array}\right\}
$$

A local semi-shock with an adjacent non-centred expansion fan occurs in the region DEFD, which separates the rarefaction fan from the layer of small particles below. 


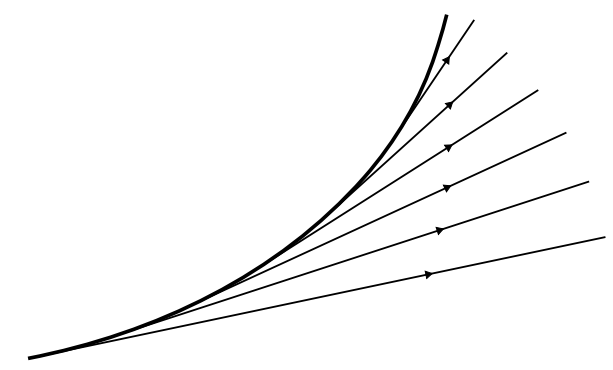

FIGURE 12. A sketch of the non-centred expansion fan arising within the region DEFD, where each characteristic of the rarefaction is tangential to the semi-shock.

Each incoming characteristic from the rarefaction fan (6.2) intersects semi-shock DE and generates a new characteristic that lies locally tangent to it. The semi-shock DE therefore separates each rarefaction characteristic $\phi_{+}=\phi$ from its image point concentration $\phi_{-}=\phi^{\circ}$. Using the chain rule and the definition of the image point (3.6), the shock gradient (4.9) can be solved implicitly by differentiating the expansion fan characteristics (6.2) with respect to $\phi$, to obtain a separable ODE for the semi-shock DE, i.e.

$$
\frac{1}{\xi} \frac{\mathrm{d} \xi}{\mathrm{d} \phi}=\frac{F^{\prime \prime}(\phi)}{F^{\prime}\left(\phi^{\circ}\right)-F^{\prime}(\phi)} .
$$

For a general flux function,

$$
\frac{\mathrm{d}}{\mathrm{d} \phi}\left(F^{\prime}\left(\phi^{\circ}\right)-F^{\prime}(\phi)\right)=F^{\prime \prime}\left(\phi^{\circ}\right) \frac{\mathrm{d} \phi^{\circ}}{\mathrm{d} \phi}-F^{\prime \prime}(\phi),
$$

but, since $F^{\prime \prime}\left(\phi^{\circ}\right) \mathrm{d} \phi^{\circ} / \mathrm{d} \phi \neq 0,(6.15)$ cannot normally be integrated to give a simple logarithmic form as with (5.11), (6.5) and (6.7). Instead, (6.15) must be numerically integrated with the initial condition that the semi-shock starts at point D. However, the cubic flux function (3.4) satisfies

$$
\frac{\mathrm{d}}{\mathrm{d} \phi}\left(F^{\prime}\left(\phi^{\circ}\right)-F^{\prime}(\phi)\right)=\frac{\mathrm{d}}{\mathrm{d} \phi}\left(-\frac{1}{4 \gamma}(3 \gamma \phi-(1+\gamma))^{2}\right)=-\frac{3}{4} F^{\prime \prime}(\phi),
$$

and so (6.15) may be integrated exactly. As semi-shock DE starts from point $\mathrm{D}$, it has the implicit coordinates $\left(\xi_{\mathrm{DE}}, \psi_{\mathrm{DE}}\right)$,

$$
\xi_{\mathrm{DE}}=\frac{\psi_{\mathrm{A}} \gamma^{2}}{S_{r} A_{\gamma}}\left(\frac{256}{(2 \gamma-1)(3 \gamma \phi-(1+\gamma))^{8}}\right)^{1 / 3}, \quad \psi_{\mathrm{DE}}=\psi_{\mathrm{A}}-S_{r} F^{\prime}(\phi) \xi_{\mathrm{DE}}
$$

for $\phi \in\left[\phi_{\mathrm{D}}, \phi_{\mathrm{E}}\right]$. The non-centred fan is formed from each of the $\phi^{\circ}$ characteristics that are local tangents to the semi-shock $\mathrm{DE}$ and are given by the straight lines

$$
\psi=\psi_{\mathrm{DE}}(\phi)-S_{r} F^{\prime}\left(\phi^{\circ}\right)\left(\xi-\xi_{\mathrm{DE}}(\phi)\right), \quad \text { for } \phi \in\left[\phi_{\mathrm{D}}, \phi_{\mathrm{E}}\right] .
$$

This forms the beautiful structure sketched in figure 12 .

Semi-shock DE meets the upper shock $\mathrm{CE}$ at point E. Equating $\xi_{\mathrm{DE}}=\xi_{\mathrm{CE}}$ using (6.19) and (6.6) gives both the coordinates $\left(\xi_{\mathrm{E}}, \psi_{\mathrm{E}}\right)$ and concentration $\phi_{\mathrm{E}}$. A further shock EF separates the upper layer of large particles $\left(\phi_{+}=0\right)$ from the local 

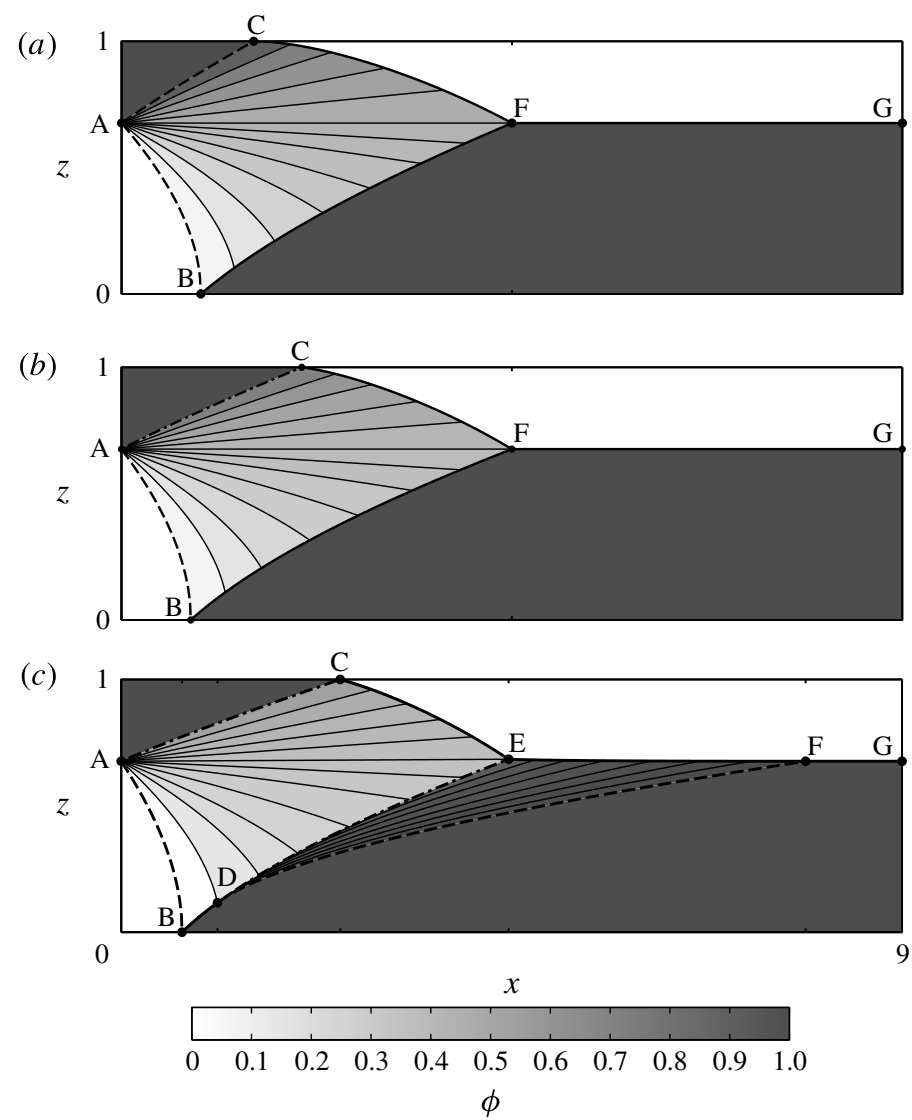

FIGURE 13. Exact solutions for an initially normally graded mixture in physical $(x, z)$ coordinates with a Bagnold velocity profile (6.34). As in figure $10, \psi_{\mathrm{A}}=0.50$ (which maps to $\left.z_{\mathrm{A}}=0.676\right), S_{r}=0.5$, and the cubic flux function (3.4) has been used with $(a) \gamma=0.35$, (b) $\gamma=0.65$ and $(c) \gamma=0.90$. Thick solid lines represent shocks, thick dash-dot lines are semi-shocks whilst thick dashed lines represent the edge of rarefaction fans. Characteristics are shown with thin solid black lines. The velocity is monotonically increasing with height, so the large-particle layer at the top is thinner to conserve mass.

rarefaction fan $\left(\phi_{-}=\phi^{\circ}\right)$. Using the chain rule, the shock gradient (4.9) can be rearranged to give

$$
\frac{\mathrm{d} \psi}{\mathrm{d} \phi^{\circ}}=-S_{r} \frac{F\left(\phi^{\circ}\right)}{\phi^{\circ}} \frac{\mathrm{d} \xi}{\mathrm{d} \phi^{\circ}}
$$

whilst the rarefaction fan characteristics (6.19) may be differentiated implicitly with respect to $\phi^{\circ}$ to give

$$
\frac{\mathrm{d} \psi}{\mathrm{d} \phi^{\circ}}-\frac{\mathrm{d} \psi_{\mathrm{DE}}}{\mathrm{d} \phi^{\circ}}=-S_{r} \frac{\mathrm{d}^{2} F\left(\phi^{\circ}\right)}{\mathrm{d}\left(\phi^{\circ}\right)^{2}}\left(\xi-\xi_{\mathrm{DE}}\right)-S_{r} \frac{\mathrm{d} F\left(\phi^{\circ}\right)}{\mathrm{d} \phi^{\circ}}\left(\frac{\mathrm{d} \xi}{\mathrm{d} \phi^{\circ}}-\frac{\mathrm{d} \xi_{\mathrm{DE}}}{\mathrm{d} \phi^{\circ}}\right) .
$$

By combining (6.20) and (6.21), the coordinates of EF can be shown to satisfy the inhomogeneous differential equation

$$
-\frac{\mathrm{d}}{\mathrm{d} \phi^{\circ}}\left[\left(F\left(\phi^{\circ}\right)-\phi^{\circ} F^{\prime}\left(\phi^{\circ}\right)\right) \xi\right]=\phi^{\circ} \frac{\mathrm{d}}{\mathrm{d} \phi^{\circ}}\left(\frac{\psi_{\mathrm{DE}}(\phi)}{S_{r}}+F^{\prime}\left(\phi^{\circ}\right) \xi_{\mathrm{DE}}(\phi)\right) .
$$


The image point concentration $\phi^{\circ}$ has been treated as the independent variable, with the relationship between $\phi$ and $\phi^{\circ}$ governed by (3.6). Equation (6.22) is a first-order differential equation of the form

$$
-\frac{\mathrm{d}}{\mathrm{d} \phi^{\circ}}\left(f\left(\phi^{\circ}\right) \xi\right)=\phi^{\circ} \frac{\mathrm{d}}{\mathrm{d} \phi^{\circ}} g\left(\phi^{\circ}, \phi\right),
$$

and since (6.18) expresses DE exactly for the cubic flux function, (6.22) can be integrated by parts with the boundary condition that $\phi=\phi_{\mathrm{E}}, \phi^{\circ}=\phi_{\mathrm{E}}^{\circ}$ at $\xi=\xi_{\mathrm{E}}$. This gives the implicit coordinates $\left(\xi_{\mathrm{EF}}(\phi), \psi_{\mathrm{EF}}(\phi)\right)$ of $\mathrm{EF}$ as

$$
\left.\begin{array}{l}
\xi_{\mathrm{EF}}(\phi)=\frac{g_{1}\left(\phi_{\mathrm{E}}\right) \xi_{\mathrm{E}}+g_{2}(\phi)-g_{2}\left(\phi_{\mathrm{E}}\right)}{g_{1}(\phi)}, \\
\psi_{\mathrm{EF}}(\phi)=\psi_{\mathrm{DE}}(\phi)-S_{r} F^{\prime}\left(\phi^{\circ}\right)\left(\xi_{\mathrm{EF}}(\phi)-\xi_{\mathrm{DE}}(\phi)\right),
\end{array}\right\}
$$

where $\phi_{\mathrm{D}} \leqslant \phi \leqslant \phi_{\mathrm{E}}$ and the functions $g_{1}(u)$ and $g_{2}(u)$ are defined as

$$
g_{1}(u)=\frac{A_{\gamma}}{4 \gamma} u(1+\gamma-\gamma u)^{2}, \quad g_{2}(u)=\frac{\psi_{\mathrm{A}} \gamma}{S_{r}}\left(\frac{4}{2 \gamma-1}\right)^{1 / 3} u(3 \gamma u-(1+\gamma))^{-2 / 3} .
$$

The $\phi=1$ characteristic that is tangential at point $\mathrm{D}$ meets the upper layer at point $\mathrm{F}$, whose coordinates $\left(\xi_{\mathrm{F}}, \psi_{\mathrm{F}}\right)$ are given by $(6.24)$ with $\phi=\phi_{\mathrm{D}}$. A final shock FG separates the final inversely graded flow, with coordinates $\left(\xi_{\mathrm{FG}}, \psi_{\mathrm{FG}}\right)$ given by $(6.11)$.

The change between the two non-convex structures (figure 10b,c) occurs when point $\mathrm{D}$ coincides with point $\mathrm{E}$ and point $\mathrm{F}$. For the cubic flux function, equating $\psi_{\mathrm{F}}(6.10)$ with $\psi_{\mathrm{D}}(6.14)$ determines the curve in figure 11 as

$$
\psi_{\mathrm{A}}=\frac{8 \gamma^{3}-12 \gamma^{2}+6 \gamma-1}{11 \gamma^{3}-19 \gamma^{2}+11 \gamma-2} .
$$

\subsection{Comparison with the symmetric model}

The asymmetric flux functions lead to several differences in the solution for normally graded inflow that are not found in the structure for the quadratic flux derived by Thornton et al. (2006). Firstly, although the convex flux structure shown in figure 10(a) appears similar to that of the convex quadratic flux (2.14), the positions of points $\mathrm{B}$ and $\mathrm{C}$ are modified due to the particles percolating downwards and rising upwards at different rates. Thornton et al. (2006) found the ratio of $\xi_{\mathrm{C}}$ to $\xi_{\mathrm{B}}$ to be dependent on the initial discontinuity height $\psi_{\mathrm{A}}$, i.e.

$$
\frac{\xi_{\mathrm{C}}}{\xi_{\mathrm{B}}}=\frac{\left(1-\psi_{\mathrm{A}}\right)}{\psi_{\mathrm{A}}},
$$

but, using (6.3) and (6.4), for convex flux functions,

$$
\frac{\xi_{\mathrm{C}}}{\xi_{\mathrm{B}}}=-\frac{F^{\prime}(0)}{F^{\prime}(1)} \frac{\left(1-\psi_{\mathrm{A}}\right)}{\psi_{\mathrm{A}}}=\frac{1}{(1-\gamma)} \frac{\left(1-\psi_{\mathrm{A}}\right)}{\psi_{\mathrm{A}}},
$$

which, for the cubic flux function (3.4), has an additional dependence on $\gamma$. In the limit as $\gamma \rightarrow 0$ the quadratic result is recovered. This asymmetry is also seen with 
the non-convex flux functions in figure $10(b, c)$, but now several large particles rise together at concentration $\phi_{c r i t}=\phi_{\mathrm{C}}$ and reach the top at C. From (6.13), this gives

$$
\frac{\xi_{\mathrm{C}}}{\xi_{\mathrm{B}}}=-\frac{F^{\prime}(0)}{F^{\prime}\left(\phi_{\mathrm{C}}\right)} \frac{\left(1-\psi_{\mathrm{A}}\right)}{\psi_{\mathrm{A}}}=4 \gamma \frac{\left(1-\psi_{\mathrm{A}}\right)}{\psi_{\mathrm{A}}} .
$$

The ratio $\xi_{\mathrm{C}} / \xi_{\mathrm{B}}$ given by (6.28) and (6.29) could provide a sensitive experimental test for measuring the asymmetry between the maximum large- and small-particle velocities. For example, consider run 22 in the chute flow experiment of Wiederseiner et al. (2011), with $z_{\mathrm{A}}=0.7$. The velocity data were found to fit an exponential velocity profile

$$
u=\frac{\beta \exp (\beta z)}{\exp (\beta)-1},
$$

with $\beta=3.3$, which maps $z_{\mathrm{A}}$ to $\psi_{\mathrm{A}}=0.374$ through transformation (4.2). Using the $\phi=0$ contour in figure 7(a) of their paper, it is possible to estimate $\xi_{\mathrm{B}}=0.17$ and $\xi_{\mathrm{C}}=0.53$, which give

$$
\frac{\xi_{\mathrm{C}} / \xi_{\mathrm{B}}}{\left(1-\psi_{\mathrm{A}}\right) / \psi_{\mathrm{A}}}=1.86
$$

Note that this ratio is greater than the ratio 1 of the quadratic flux (6.27), but less than 2. Assuming the cubic flux (3.4), comparing (6.31) with (6.28) implies that

$$
\gamma=0.46
$$

A second difference between the quadratic flux model and the asymmetric flux functions is the presence of semi-shocks and adjacent expansion fans, such as DEFD in figure $10(c)$ for non-convex flux functions. A similar semi-shock and adjacent fan AEFA also develops in the homogeneous case in figure $7(b, c)$. In both cases, these are a direct result of the decreasing rise velocity of large particles above concentration $\phi=\phi_{\mathrm{C}}$.

\subsection{Solution in physical coordinates}

The solution in physical coordinates $(x, z)$ may easily be derived from the mapped coordinates $(\xi, \psi)$ by prescribing a downslope velocity field $u=u(z)$ and inverting the coordinate transformation (4.2). A Bagnold velocity profile typically develops for steady uniform flows (e.g Bagnold 1954; Silbert et al. 2001; GDR MiDi 2004; Rognon et al. 2007), which in dimensional variables is

$$
u=\frac{2}{3} \frac{I_{\zeta}}{d} \sqrt{g \Phi \cos \zeta}\left(h^{3 / 2}-(h-z)^{3 / 2}\right),
$$

where $I_{\zeta}$ is the constant inertial number at a given inclination angle $\zeta$ (GDR MiDi 2004; Jop et al. 2005; Gray \& Edwards 2014), $\Phi$ is the solids volume fraction and $d$ is a measure of the average diameter of the grains. Applying the non-dimensionalisation (2.17), with the velocity magnitude $U$ chosen so that $\psi(1)=1$, gives the non-dimensional downstream velocity

$$
u(z)=\frac{5}{3}\left(1-(1-z)^{3 / 2}\right) .
$$

The transformation to mapped coordinates (4.2) gives

$$
\psi=\frac{5}{3} z-\frac{2}{3}\left(1-(1-z)^{5 / 2}\right) .
$$


This transformation cannot be inverted to produce an explicit function for $z(\psi)$; however, contour plots of the results are easy to produce by numerically inverting the normal coordinate, and are shown in figure 13. The inverse transformation effectively stretches the vertical coordinate, and transforms straight lines in mapped $(\xi, \psi)$ coordinates to curves in physical $(x, z)$ coordinates. Shock FG is the only line to remain straight after the transformation, but it is translated to a new height $z\left(1-\psi_{\mathrm{A}}\right)$. The velocity is monotonically increasing towards the surface, and thus there is also an increasing mass flux with height. In order to conserve mass, layers in regions of high velocity near the surface will be thinner than layers in regions of lower velocity near the base. Hence, for the example shown in figure 10, the layer of large particles at the top is the same thickness as the layer of small particles at the bottom in $(\xi, \psi)$ space. After the transformation to physical coordinates, the large-particle layer at the surface is thinner, whilst the small-particle layer is expanded. The transformation leaves the downstream coordinate unchanged, and so horizontal coordinates of points $\mathrm{A}-\mathrm{G}$ and the final segregation distances are left unaffected.

\section{Discussion and conclusions}

This paper generalises the particle-size segregation model of Gray \& Thornton (2005) to asymmetric flux functions, in order to model the observation that a single small particle will percolate down through a matrix of large particles faster than a single large grain will rise up through a matrix of fines. A general class of asymmetric flux functions is considered, whose maximum amplitude is skewed towards lower concentrations of fines, and which may be either convex or non-convex, as shown for the cubic case in figure 3. For convex flux functions, the maximum percolation velocity of the fines, which occurs in the limit as the concentration of fines tends to $0 \%$, is enhanced above that obtained with the simple quadratic flux used by Gray \& Thornton (2005). Conversely, the maximum rise rate of large particles occurs in the limit of $100 \%$ fines, and is decreased from that of the quadratic case, as shown in figure 4. For non-convex flux functions, the maximum rise rate of large particles occurs at an intermediate concentration, which directly leads to the formation of more complex solutions that include semi-shocks and non-centred expansion fans, as well as shocks and centred fans.

In the limit of no diffusive remixing, the method of characteristics is used to derive exact steady-state concentration solutions for the homogeneous and normally graded inflow problems of Gray \& Thornton (2005) and Thornton et al. (2006). The results are illustrated for the case of the cubic flux function in figures 7, 10 and 13. In each case, there are three qualitatively different forms of the solution, which are dependent on the inflow concentration distribution and whether the flux function is convex or non-convex. The parameter dependence of the solutions is illustrated in figures 8 and 11. For convex flux functions, the solutions look very similar to those constructed by Gray \& Thornton (2005) and Thornton et al. (2006), except that the position of key points in the solution are now dependent on the inflow composition and the strength of asymmetry. In particular, the asymmetry causes the final segregation distance for homogeneous flow to be dependent on the inflow concentration (5.14), and in the normally graded problem the ratio of the positions for the first large particle to reach the surface and the first small particle to reach the base, (6.28) and (6.29), is dependent on the skewness. Comparing the theory to the experiments of Wiederseiner et al. (2011) suggests that $\gamma=0.46$, which is close to the boundary between convex and non-convex flux functions. For non-convex 
flux functions, semi-shocks with adjacent centred rarefaction fans (such as AEFA in figure 7) and non-centred rarefaction fans (such as DEFD in figure 10) appear, which will also not be completely smeared out by diffusive remixing.

The asymmetric theory of segregation analysed in this paper can easily be extended to account for multi- or polydisperse mixtures (Gray \& Ancey 2011; Marks et al. 2012), and flows that include erosion and deposition (Gray \& Ancey 2009; Fan et al. 2014). Although a cubic form has been used in this paper to illustrate the effects of an asymmetric flux function, the actual flux function may also take a more complicated form, potentially depending on a number of flow parameters (Bridgwater 1994). For example, the amount of asymmetry is likely to be physically dependent on the size ratio of the particles, with similar sized particles displaying less asymmetry, but more asymmetry experienced as the spontaneous percolation limit is approached (Bridgwater \& Ingram 1971; Savage \& Lun 1988). However, this is still to be experimentally verified, and the actual functional form for the segregation flux and its parameter dependences are open questions.

\section{Acknowledgements}

The authors would like to thank K. van der Vaart for many enlightening discussions. This research was supported by NERC and EPSRC grants NE/K003011/1 and EP/K00428X/1. P.G. also acknowledges support from the Margaret Elizabeth Lee Fellowship, and J.M.N.T.G. acknowledges support from the programme on 'Fluid-Mediated Particle Transport in Geophysical Flows' at the Kavli Institute for Theoretical Physics, Santa Barbara, CA, USA.

\section{REFERENCES}

BAgnOLD, R. A. 1954 Experiments on gravity-free dispersion of large solid spheres in a Newtonian fluid under shear. Proc. R. Soc. Lond. A 225, 49-63.

Bartelt, P. \& McArdell, B. W. 2009 Granulometric investigations of snow avalanches. J. Glaciol. 55 (193), 829-833.

Batchelor, G. K. 1972 Sedimentation in a dilute dispersion of spheres. J. Fluid Mech. 52, 245-268.

Berryman, J. G. 1983 Random close packing of hard spheres and disks. Phys. Rev. A 27, 1053-1061.

Branney, M. J. \& KoKelaAR, B. P. 1992 A reappraisal of ignimbrite emplacement: progressive aggradation and changes from particulate to non-particulate flow during emplacement of highgrade ignimbrite. Bull. Volcanol. 54, 504-520.

BRIDGWATER, J. 1994 Mixing and segregation mechanisms in particle flow. In Granular Matter (ed. A. Mehta), pp. 161-193. Springer.

Bridgwater, J., Foo, W. \& StePhens, D. 1985 Particle mixing and segregation in failure zones theory and experiment. Powder Technol. 41, 147-158.

Bridgwater, J. \& IngRAM, N. D. 1971 Rate of spontaneous inter-particle percolation. Trans. Inst. Chem. Engrs 49 (3), 163-169.

Buckley, S. E. \& Leverett, M. C. 1942 Mechanism of fluid displacement in sands. Trans. AIME 146, $107-116$.

Calder, E. S., Sparks, R. S. J. \& Gardeweg, M. C. 2000 Erosion, transport and segregation of pumice and lithic clasts in pyroclastic flows inferred from ignimbrite at Lascar volcano, Chile. J. Volcanol. Geotherm. Res. 104, 201-235.

Chadwick, P. 1976 Continuum Mechanics. Concise Theory and Problems. George Allen \& Unwin.

Courant, R. \& Hilbert, D. 1962 Methods of Mathematical Physics, vol. II. Interscience.

DAsGupta, P. \& MAnna, P. 2011 Geometrical mechanism of inverse grading in grain-flow deposits: an experimental revelation. Earth-Sci. Rev. 104 (1-3), 186-198. 
Dingler, J. R. \& Anima, R. J. 1987 Subaqueous grain flows at the head of Carmel submarine canyon, California. J. Sedim. Petrol. 59 (2), 280-286.

Dolgunin, V. N. \& UKOlov, A. A. 1995 Segregation modelling of particle rapid gravity flow. Powder Technol. 83, 95-103.

DYER, F. C. 1929 The scope for reverse classification by crowded settling in ore-dressing practice. Eng. Min. J. 127 (26), 1030-1033.

Fan, Y., Boukerkour, Y., Blanc, T., Umbanhowar, P. B., Ottino, J. M. \& Lueptow, R. M. 2012 Stratification, segregation, and mixing of granular materials in quasi-two-dimensional bounded heaps. Phys. Rev. E 86, 051305.

FAN, Y. I. \& HILL, K. M. 2011 Theory for shear-induced segregation of dense granular mixtures. New J. Phys. 13 (9), 095009.

Fan, Y., Schlick, C. P., Umbanhowar, P. B., Ottino, J. M. \& Lueptow, R. M. 2014 Modelling size segregation of granular materials: the roles of segregation, advection and diffusion. J. Fluid Mech. 741, 252-279.

Fisher, R. V. \& MATtinson, J. M. 1968 Wheeler gorge turbidite-conglomerate series California inverse grading. J. Sedim. Petrol. 38 (4), 1013-1023.

Forterre, Y. \& Pouliquen, O. 2008 Flows of dense granular media. Annu. Rev. Fluid Mech. 40 (1), 1-24.

GDR MiDi 2004 On dense granular flows. Eur. Phys. J. E 14, 341-365.

Golick, L. A. \& Daniels, K. E. 2009 Mixing and segregation rates in sheared granular materials. Phys. Rev. E 80 (4), 042301.

GraY, J. M. N. T. 2001 Granular flow in partially filled slowly rotating drums. J. Fluid Mech. 441, 1-29.

Gray, J. M. N. T. \& ANCEY, C. 2009 Segregation, recirculation and deposition of coarse particles near two-dimensional avalanche fronts. J. Fluid Mech. 629, 387-423.

Gray, J. M. N. T. \& ANCEY, C. 2011 Multi-component particle-size segregation in shallow granular avalanches. J. Fluid Mech. 678, 535-588.

Gray, J. M. N. T. \& Chugunov, V. A. 2006 Particle-size segregation and diffusive remixing in shallow granular avalanches. J. Fluid Mech. 569, 365-398.

Gray, J. M. N. T. \& EDWARDS, A. N. 2014 A depth-averaged $\mu(I)$-rheology for shallow granular free-surface flows. J. Fluid Mech. 755, 503-534.

Gray, J. M. N. T. \& Hutter, K. 1997 Pattern formation in granular avalanches. Contin. Mech. Thermodyn. 9, 341-345.

Gray, J. M. N. T. \& KokelaAR, B. P. $2010 a$ Large particle segregation, transport and accumulation in granular free-surface flows. J. Fluid Mech. 652, 105-137.

Gray, J. M. N. T. \& KoKelaAR, B. P. $2010 b$ Large particle segregation, transport and accumulation in granular free-surface flows - Erratum. J. Fluid Mech. 657, 539.

Gray, J. M. N. T. \& Thornton, A. R. 2005 A theory for particle size segregation in shallow granular free-surface flows. Proc. R. Soc. Lond. A 461, 1447-1473.

Gray, J. M. N. T., Wieland, M. \& Hutter, K. 1999 Free surface flow of cohesionless granular avalanches over complex basal topography. Proc. R. Soc. Lond. A 455, 1841-1874.

Greenberg, H. 1959 An analysis of traffic flow. Oper. Res. 7 (1), 79-85.

Hill, K. M., Kharkar, D. V., Gilchrist, J. F., McCarthy, J. J. \& Ottino, J. M. 1999 Segregation driven organization in chaotic granular flows. Proc. Natl Acad. Sci. USA 96, 11701-11706.

Hutter, K., WAng, Y. Q. \& Pudasaini, S. P. 2005 The Savage-Hutter avalanche model: how far can it be pushed?. Phil. Trans. R. Soc. Lond. A 363 (1832), 1507-1528.

IVERSOn, R. M. 1997 The physics of debris-flows. Rev. Geophys. 35, 245-296.

IVERSON, R. M. \& VAllance, J. W. 2001 New views of granular mass flows. Geology 29 (2), $115-118$.

Jeffrey, A. 1976 Quasilinear Hyperbolic Systems and Waves. Pitman.

Johanson, J. R. 1978 Particle segregation ... and what to do about it. Chem. Engng, 8 May, pp. 183-188. 
Johnson, C. G., Kokelaar, B. P., Iverson, R. M., Logan, M., LaHusen, R. G. \& GRAY, J. M. N. T. 2012 Grain-size segregation and levee formation in geophysical mass flows. J. Geophys. Res. 117, F01032.

Jop, P., Forterre, Y. \& Pouliquen, O. 2005 Crucial role of sidewalls in granular surface flows: consequences for the rheology. J. Fluid Mech. 541, 167-192.

Khakhar, D. V., MCCARthy, J. J. \& OtTino, J. M. 1999 Mixing and segregation of granular materials in chute flows. Chaos 9 (3), 594-610.

Kokelaar, B. P., Graham, R. L., Gray, J. M. N. T. \& Vallance, J. W. 2014 Fine-grained linings of leveed channels facilitate runout of granular flows. Earth Planet. Sci. Lett. 385, $172-180$.

Kumaran, V. 2006 The constitutive relation for the granular flow of rough particles, and its application to the flow down an inclined plane. J. Fluid Mech. 561, 1-42.

Kumaran, V. 2008 Dense granular flow down an inclined plane: from kinetic theory to granular dynamics. J. Fluid Mech. 599, 121-168.

KYNCH, G. J. 1952 A theory of sedimentation. Trans. Faraday Soc. 48, 166-176.

Laney, C. B. 1998 Computational Gasdynamics. Cambridge University Press.

LAX, P. D. 1957 Hyperbolic systems of conservation laws II. Commun. Pure Appl. Maths 10 (4), 537-566.

Lighthill, M. J. \& Whitham, G. B. 1955 On kinematic waves: II. A theory of traffic flow on long crowded roads. Proc. R. Soc. Lond. A 229 (1178), 317-345.

LiU, T. P. 1974 The Riemann problem for general $2 \times 2$ conservation laws. Trans. Am. Math. Soc. 199, 89-112.

MARKS, B. \& EINAV, I. 2011 A cellular automaton for segregation during granular avalanches. Granul. Matt. 13 (3), 211-214.

Marks, B., Rognon, P. \& EinAV, I. 2012 Grainsize dynamics of polydisperse granular segregation down inclined planes. J. Fluid Mech. 690, 499-511.

Marks, B., Valaulta, A., Puzrin, A. \& EinaV, I. 2013 Design of protection structures: the role of the grainsize distribution. In Powders and Grains 2013: Proceedings of the 7th International Conference on Micromechanics of Granular Media, Sydney, Australia, 8-12 July (ed. A. Yu, K. Dong, R. Yang \& S. Luding), AIP Conference Proceedings, vol. 1542, pp. 658-661. American Institute of Physics.

May, L. B. H., Golick, L. A., Phillips, K. C., Shearer, M. \& Daniels, K. E. $2010 a$ Shear-driven size segregation of granular materials: modeling and experiment. Phys. Rev. E 81, 051301.

May, L. B. H., Shearer, M. \& DAniels, K. E. $2010 b$ Scalar conservation laws with non-constant coefficients with application to particle size segregation in granular flow. J. Nonlinear Sci. 20 (6), 689-707.

Middleton, G. V. 1970 Experimental studies related to problems of flysch sedimentation. In Flysch Sedimentology in North America (ed. J. Lajoie), Geological Association of Canada, Special paper 7, pp. 253-272. Business and Economic Service.

Morland, L. W. 1992 Flow of viscous fluids through a porous deformable matrix. Surv. Geophys. 13 (3), 209-268.

Nityanand, N., Manley, B. \& Henein, H. 1986 An analysis of radial segregation for different sized spherical solids in rotary cylinders. Metall. Trans. B 17 (2), 247-257.

OlEINIK, O. A. 1959 Uniqueness and stability of the generalized solution of the Cauchy problem for a quasi-linear equation. Usp. Mat. Nauk (NS) 14, 165-170.

Ottino, J. M. \& KhakhaR, D. V. 2000 Mixing and segregation of granular materials. Annu. Rev. Fluid Mech. 32 (1), 55-91.

Pitman, E. B., Nichita, C. C., Patra, A., Bauer, A., Sheridan, M. \& Bursik, M. 2003 Computing granular avalanches and landslides. Phys. Fluids 15 (12), 3638-3646.

Pouliquen, O. 1999 Scaling laws in granular flows down rough inclined planes. Phys. Fluids 11 (3), 542-548. 
Pudasaini, S. P. \& Hutter, K. 2007 Avalanche Dynamics: Dynamics of Rapid Flows of Dense Granular Avalanches. Springer.

Rhee, H. K., Aris, R. \& Amundson, N. R. 1986 First-order Partial Differential Equations, Theory and Applications of Single Equations, vol. 1. Prentice-Hall.

Rognon, P. G., Roux, J. N., NAAim, M. \& Chevoir, F. 2007 Dense flows of bidisperse assemblies of disks down an inclined plane. Phys. Fluids 19 (5), 058101.

SAVAgE, S. B. \& HUtTER, K. 1989 The motion of a finite mass of granular material down a rough incline. J. Fluid Mech. 199, 177-215.

Savage, S. B. \& Lun, C. K. K. 1988 Particle size segregation in inclined chute flow of dry cohesionless granular solids. J. Fluid Mech. 189, 311-335.

SCHMinck, H. U. 1967 Graded lahars in type sections of Ellensburg Formation, south-central Washington. J. Sedim. Petrol. 37 (2), 438-448.

Shannon, P. T., Stroupe, E. \& Tory, E. M. 1963 Batch and continuous thickening - basic theory - solids flux for rigid spheres. Ind. Engng Chem. Fundam. 2 (3), 203-211.

Shinbrot, T. \& Muzzio, F. J. 2000 Non-equilibrium patterns in granular mixing and segregation. Phys. Today 53 (3), 25-30.

Silbert, L. E., Ertas, D., Grest, G. S., Halsey, T. C., Levine, D. \& Plimpton, S. J. 2001 Granular flow down an inclined plane: Bagnold scaling and rheology. Phys. Rev. E 64 (5), 051302.

Sohn, Y. K. \& Chough, S. K. 1993 The Udo tuff cone, Cheju Island, South Korea: transformation of pyroclastic fall into debris fall and grain flow on a steep volcanic cone slope. Sedimentology 40 (4), 769-786.

Stock, J. D. \& Dietrich, W. E. 2006 Erosion of steepland valleys by debris flows. Geol. Soc. Am. Bull. 118, 1125-1148.

Thornton, A. R. \& Gray, J. M. N. T. 2008 Breaking size-segregation waves and particle recirculation in granular avalanches. J. Fluid Mech. 596, 261-284.

Thornton, A. R., Gray, J. M. N. T. \& HogG, A. J. 2006 A three-phase mixture theory for particle size segregation in shallow granular free-surface flows. J. Fluid Mech. 550, 1-25.

Thornton, A. R., Weinhart, T., Luding, S. \& Bokhove, O. 2012 Modeling of particle size segregation: calibration using the discrete particle method. Intl J. Mod. Phys. C 23 (8), 1240014.

Tripathi, A. \& KhaKhaR, D. V. 2011 Rheology of binary granular mixtures in the dense flow regime. Phys. Fluids 23, 113302.

TUCKER, M. E. 2003 Sedimentary Rocks in the Field. Wiley.

Vallance, J. W. \& Savage, S. B. 2000 Particle segregation in granular flows down chutes. In IUTAM Symposium on Segregation in Granular Materials (ed. A. D. Rosato \& D. L. Blackmore), Kluwer.

Whitham, G. B. 1974 Linear and Nonlinear Waves. Wiley.

Wiederseiner, S., Andreini, N., Epely-Chauvin, G., Moser, G., Monnereau, M., GRAY, J. M. N. T. \& ANCEY, C. 2011 Experimental investigation into segregating granular flows down chutes. Phys. Fluids 23, 013301.

Williams, J. C. 1976 The segregation of particulate materials - review. Powder Technol. 15 (2), 245-251.

Woodhouse, M. J., Thornton, A. R., Johnson, C. G., KokelaAr, B. P. \& Gray, J. M. N. T. 2012 Segregation-induced fingering instabilities in granular free-surface flows. J. Fluid Mech. 709, 543-580. 